\title{
Bell inequalities for Continuous-Variable Measurements
}

\author{
Q. Y. He, ${ }^{1}$ E. G. Cavalcanti, ${ }^{2}$ M. D. Reid, ${ }^{1}$ and P. D. Drummond ${ }^{1}$ \\ ${ }^{1}$ ARC Centre of Excellence for Quantum-Atom Optics, \\ Swinburne University of Technology, Melbourne, Australia. \\ ${ }^{2}$ Centre for Quantum Dynamics, Griffith University, Brisbane QLD 4111, Australia
}

\begin{abstract}
Tests of local hidden variable theories using measurements with continuous variable (CV) outcomes are developed, and a comparison of different methods is presented. As examples, we focus on multipartite entangled GHZ and cluster states. We suggest a physical process that produces the states proposed here, and investigate experiments both with and without binning of the continuous variable. In the former case, the Mermin-Klyshko inequalities can be used directly. For unbinned outcomes, the moment-based CFRD inequalities are extended to functional inequalities by considering arbitrary functions of the measurements at each site. By optimising these functions, we obtain more robust violations of local hidden variable theories than with either binning or moments. Recent inequalities based on the algebra of quaternions and octonions are compared with these methods. Since the prime advantage of CV experiments is to provide a route to highly efficient detection via homodyne measurements, we analyse the effect of noise and detection losses in both binned and unbinned cases. The CV moment inequalities with an optimal function have greater robustness to both loss and noise. This could permit a loophole-free test of Bell inequalities.
\end{abstract}

\section{INTRODUCTION}

In 1964 Bell showed that the predictions of quantum mechanics are not compatible with those of local hidden variable theories (LHV) [1]. Since then, there have been experimental tests [2-4] of these predictions, which can be used to rule out some LHV alternatives to quantum mechanics (QM). While experiments to date support quantum mechanics, the experimental strategies used have loopholes [5]. In some experiments, problems are caused by insufficient spatial separation of measurements [6] - so that the assumption of local causality that underpins the LHV model cannot be applied to start with. In other experiments, the poor photon detection efficiencies [7] mean that correlations are not strong enough to violate a Bell inequality, unless additional assumptions are utilized. In short, a clear violation applicable to all local hidden variable theories has not yet been experimentally observed.

It has been widely suggested that the use of highly efficient detection techniques like homodyne detection [8] may provide an avenue to overcome the detection efficiency problems [5] with Bell's theorem. Already, continuous variable $(\mathrm{CV})$ realizations of the Einstein-PodolskyRosen (EPR) paradox have been achieved with high efficiencies [9], using optical homodyne detection [10]. In this case, the EPR paradox is reformulated in terms of field quadrature phase amplitudes which have commutators like position and momentum. The EPR paradox, however, only demonstrates the incompatibility of local realism (LR) with the completeness of quantum mechanics. It does not test local realism itself. Proposals to demonstrate an incompatibility of quantum theory with local hidden variable theories using continuous variables require new formalisms, since Bell's original inequality was only applicable to discrete or binned outcomes.

Early proposals for such tests using CV measurements [8] relied on binning the initially continuous outcome do- mains to obtain binary results. Here the original Bell Clauser-Horne-Shimony-Holt inequalities [11] are applicable. However, these predicted violations of Bell's inequality were small and involved states that were difficult to prepare. Wenger et al. advocated a root binning technique to enhance the level of violation [12]. Nha and Carmichael [13] proposed a conditional state preparation to obtain the required nonclassical states. Munro et al. [14] and Acin et al. [15] suggested using binned $\mathrm{CV}$ measurements made on $N$ spatially separated qubit systems prepared in a Greenberger-HorneZeilinger (GHZ) state [16], and noted improved violation of the Mermin-Ardehali-Belinskii-Klyshko (MABK) Bell inequalities [17] as $N$ is increased.

More recently, Cavalcanti, Foster, Reid and Drummond (CFRD) [18] have derived a continuous variable inequality for LHV theories, formulated directly in terms of moments, without binning. This allows a simple test of local hidden variable theories for genuine $\mathrm{CV}$ outcomes. Violations of this CV moment inequality were found possible for GHZ states involving a large number of sites $(N \geq 10)$. Based on the CFRD inequalities, Shchukin and Vogel [19] introduced further inequalities considering 4 or 8 observables at each site. Salles et al have shown[20] that at least three sites are necessary for the CFRD inequalities to be violated, and have more recently generalized this technique to multilinear mappings[21]. In general, the use of ordinary moments has the advantage that it is more robust against loss and noise than earlier binning or conditional techniques. However, the large number of multipartite sites involved presents practical difficulties.

In this paper, we develop functional CFRD Bell inequalities for testing local hidden variable theories, using arbitrary functions of continuous variable outcomes at each site. Variational calculus is used to optimize the choice of measured function, to maximise the violation of the Bell inequality. As a result, we obtain predictions for 
violations of LHV models with fewer sites, larger losses and greater degrees of decoherence than previously possible. This approach of optimising the function of a measurement result for a particular purpose should be generally useful in the field of quantum information. Preliminary results have been presented as a short Letter [22]. Here we provide a more detailed and thorough account of this procedure.

The work of Acin et al. [15] and Cavalcanti et al. [18] has revealed an exponential improvement in Bell inequality violations as the number of sites $N$ increases, for $\mathrm{CV}$ measurements. These results support the earlier work of MABK, which also showed an exponential improvement of violation for $N$-partite states for spin measurements. Since these inequalities require detection at every site, one might expect this improvement to be counteracted by increasing sensitivity to detection losses. Work on the robustness of Mermin-Bell and CFRD-Bell violations with loss [18, 24] shows that this is not the case. Other calculations using different LHV assumptions [25] have suggested a loss threshold as low as $\eta_{\text {crit }}=0.5$ as $N \rightarrow \infty$. However, since this proposal uses additional assumptions about the LHV models, it may not rule out all LHV theories.

In the present paper, we introduce a new approach to nonlocality that utilizes a general functional optimization of continuous variable observables. We find the optimal function that maximises a violation of the $\mathrm{CV}$ Bell inequality for a given efficiency $\eta$ and state purity $p$. We show that the optimal function has the form $x /\left(1+\varepsilon_{N} x^{2}\right)$, where $\varepsilon_{N}$ is a parameter related to $N$ and $\eta$. This gives an inequality for all LHV models, which is violated by the GHZ states of (2.3) for $N \geq 5$. The violation increases exponentially with $N$. For a pure state the loss threshold $\eta_{\text {crit }}$ decreases asymptotically to $\eta_{\infty}=0.69$ as $N \rightarrow \infty$, in the most symmetric case. Thus both the number of modes required, and the required efficiency are dramatically reduced. The wider range of quantum states considered here include states which can be straightforwardly obtained from a polarisation GHZ state of the type generated in current experiments. These are different to the extreme photon-number correlated states considered by Acin et al, which may be an experimental advantage.

A simple example of a functional transformation is obtained when binning of a CV observable is used to give discrete binary outcomes [8]. In this case, the CFRD inequalities reduce to those of Mermin [16]. Here, our analysis is similar to that of Acin et al. [15]. For binary outcomes, one can use extensions of Mermin's inequalities [17] for the extreme photon-number correlated states $|N\rangle=\left(|0\rangle^{\otimes N}+|1\rangle^{\otimes N}\right) / \sqrt{2}$. One then obtains an exponential increase of violation of LHV with $N$, and violation for $N \geq 3$. In addition, we extend the analysis of Mermin and Acin et al. [15], and calculate results for homodyne detection for more feasible types of state. However, we find $\left(\eta p^{2}\right)_{c r i t}=2^{(1-2 N) / N} \pi$, which means that this approach is more susceptible to loss and decoherence than using functional moment inequalities. The critical efficiency for a pure state at large $N$ is $\eta=0.79$, which is substantially higher than for the optimal functional CFRD approach.

The organisation of the paper is as follows. Section II gives the general notation used for continuous variable moments, and reviews the original CFRD inequality. In Section III we review and extend the CFRD inequality for the case of quadrature measurements on generalized GHZ states. Section IV considers the multipartite binning approach that uses the MABK Bell inequalities, which was also studied recently by Acin et al. for quadrature detection. In both Sections III and IV, we consider several types of GHZ state, and also present results for cluster and W-states [26, 27]. Next, in Section V these approaches are generalized to include functions of the observables. We compute the maximum violation of the inequality for several choices of function, in the case of a generalized multipartite GHZ state. Functional transformations using an optimum function, i.e., $x /\left(1+\varepsilon_{N} x^{2}\right)$, are shown to give a substantial improvement to results obtained with the CFRD approach. In Section VI these results are compared with the Shchukin-Vogel (SV) inequality for larger numbers of observables, showing that this gives identical results to the simpler CFRD method, in the cases studied. In Section VII we include the effects of loss and noise on the violations of the functional moment inequalities and MABK inequalities. Optimizing the choice of measured function reveals a quantum nonlocality for larger losses and for greater degrees of decoherence than possible previously. Section VIII gives an overview of the results.

In summary, we examine in this paper the effect of loss and state preparation impurity on tests of local realism using functional moments. Our motivation is the fundamental challenge of developing an experimentally feasible loop-hole free test of local realism, which balances the need for a simple experimental realization with robustness against loss and noise.

\section{CONTINUOUS VARIABLE MEASUREMENTS}

Early work on Bell inequalities utilized measurements of spin, making it seem that obtaining discrete measurement outcomes was a crucial ingredient to violating a Bell inequality. In fact, this is not essential. The CFRD inequality is a multipartite test of local realism involving ANY two observables measured at each of $N$ sites, with causal separation between measurement at each site. 


\section{A. General CFRD inequality}

It was shown in [18] that the following inequality must hold for all LHV theories:

$$
\left|\left\langle\prod_{n=1}^{N}\left(x_{n}+i p_{n}\right)\right\rangle_{P}\right|^{2} \leq\left\langle\prod_{n=1}^{N}\left(x_{n}^{2}+p_{n}^{2}\right)\right\rangle_{P},
$$

where $x_{n}, p_{n}$ are the outcomes of two arbitrary measurements [23] at each site labeled $n$. The measurements are causally separated, and \langle\rangle$_{P}$ indicates an average over a set of local hidden variables $\lambda$ with probability $P(\lambda)$.

In quantum mechanics, these correlations involve operators $\hat{x}_{n}, \hat{p}_{n}$ at each site $n$, with eigenvalues $x_{n}, p_{n}$. Unlike in hidden variable theories, these inequalities can be violated for certain quantum states, if the operators do not commute. Despite the notation used, nothing is assumed about the type of measurement operators. In LHV theories, the inequality must be satisfied by measurements with any type of spectrum, discrete or continuous, and even unbounded outcomes. For this inequality to be useful, one must find states and observables that violate the inequality within quantum theory, and are obtainable in an experiment.

It is important to recall here that when $\hat{x}_{n}, \hat{p}_{n}$ are described within quantum mechanics by non-commuting operators, the corresponding physical variables are not simultaneously measurable. Thus, the $N$-fold products in the above inequality each involve a sum over $2^{N}$ distinct correlation measurements with different settings of the measurement apparata.

\section{B. Quadrature measurements}

While the inequality is valid in general, we wish to apply it to experiments in quantum or atom optics, which are known to be able to produce highly correlated, nonclassical quantum states. Operationally, we will consider the case where conventional photodetectors are replaced by a homodyne detector using a local oscillator, in order to detect field quadratures rather than photon numbers. As well as involving an intrinsically efficient experimental technique, this method is already known to be robust against loss, without additional assumptions. Although quadrature measurements are traditionally used for coherent and squeezed states, they of course can also be used for states with well-defined overall particle number. In principle, exactly the same techniques apply to any boson fields, including, for example, ultra-cold atoms.

We assume there are $N$ sites at which one can make observations, with an adjustable local oscillator phase to define local quadrature operators $\hat{x}_{n}, \hat{p}_{n}$. In the case that $\hat{x}_{n}$ and $\hat{p}_{n}$ are associated with single-mode bosonic creation and annihilation operators $\hat{a}_{n}^{\dagger}, \hat{a}_{n}$, one has $\hat{x}_{n}=\left(\hat{a}_{n}+\hat{a}_{n}^{\dagger}\right) / 2$ and $\hat{p}_{n}=i\left(\hat{a}_{n}^{\dagger}-\hat{a}_{n}\right) / 2$. These are the quadrature phase amplitudes associated with the boson annihilation operator $\hat{a}_{n}$, or for suitable choice of units, (see the Appendix) the position and momentum of a harmonic oscillator, with commutators:

$$
\left[\hat{x}_{m}, \hat{p}_{n}\right]=\frac{i}{2} \delta_{m n} .
$$

One can adjust the local oscillator phase to detect linear combinations of position and momentum. Hence, we can introduce a general quadrature phase operator:

$$
\begin{aligned}
\hat{X}_{n}^{\theta} & =\hat{x}_{n} \cos \theta+\hat{p}_{n} \sin \theta \\
& =\frac{1}{2}\left(\hat{a}_{n} e^{-i \theta}+\hat{a}_{n}^{\dagger} e^{i \theta}\right) .
\end{aligned}
$$

For continuous measurements of quadrature variables on entangled boson states, we define generalized GHZ states to be:

$$
|\psi\rangle=\left(|0\rangle^{\otimes r}|1\rangle^{\otimes N-r}+|1\rangle^{\otimes r}|0\rangle^{\otimes N-r}\right) / \sqrt{2} .
$$

Here the integer $r$ is the maximum number of modes having nonzero photon number. The critical efficiency $\eta_{\text {crit }}$ required for violation of the CFRD inequality in the symmetric case of $r=N / 2$ tends to $\eta_{\text {crit }}=0.81$, as $N \rightarrow \infty$. Quadrature measurements with local oscillators are inherently highly efficient compared to spin or photon-number measurements, with reported efficiencies of $99 \%$. However, generation losses from mode-matching can degrade the experimental efficiency, so $81 \%$ is still a challenging practical benchmark, especially with large numbers of correlated modes.

\section{Functional transformations}

Functional transformations of any output measurements are always possible experimentally. Any data file of experimental results is trivially processed into a function or set of functions of the original measured data. While this procedure is not very useful for binary results, it represents a large functional space of measurements in the continuous variable case.

In terms of hidden-variable theories, this simply means that all measured quantities $x$ can be replaced by a function $f(x)$. In quantum mechanics, this implies that all eigenvalues $e$ are replaced by $f(e)$, or in operator language, the measurement operator $\hat{x}$ is replaced by $f(\hat{x})$. The details of this procedure are explained in later sections. We will show that such functional transformations greatly reduce the number of sites and the critical efficiencies needed to violate a Bell inequality.

\section{CFRD INEQUALITY}

In this section we will review the CFRD inequality [18] as applied to quadrature measurements, and provide a more complete analysis of its violation than in the Letter that introduced it. Quadrature observables at $N$ sites are considered here, as described in the previous section. 


\section{A. Quadrature CFRD inequality}

At each site, the sign associated with $\hat{p}_{n}$ can be chosen to obtain either $\hat{x}_{n}+i \hat{p}_{n}=\hat{a}_{n}$ or $\hat{x}_{n}-i \hat{p}_{n}=\hat{a}_{n}^{\dagger}$ for each of the terms in the left hand side $(L H S)$ of (2.1). The choice of sign is important to maximise the violations for a given state. Defining a variable $s_{n} \in\{-1,1\}$ to represent this choice for each site, we denote by $A_{n}(-1)=a_{n}$ and $A_{n}(1)=a_{n}^{\dagger}$ the corresponding combination of measurements at each site. The CFRD inequality then reduces to

$$
\left|\left\langle\prod_{n=1}^{N} A_{n}\left(s_{n}\right)\right\rangle_{P}\right|^{2} \leq\left\langle\prod_{n=1}^{N}\left(x_{n}^{2}+p_{n}^{2}\right)\right\rangle_{P} .
$$

CFRD showed that within quantum theory, the symmetric state

$$
\left|\frac{N}{2}\right\rangle=\frac{1}{\sqrt{2}}\left(|0\rangle^{\otimes N / 2}|1\rangle^{\otimes N / 2}+|1\rangle^{\otimes N / 2}|0\rangle^{\otimes N / 2}\right)
$$

violates this inequality for $N \geq 10$. Note here that there is a spatial mode index that has been dropped for convenience but is given by the order of the kets, so that this state represents $N$ distinct modes at spatially separated sites, and each mode is occupied by one or zero photons. Thus, the states $|0\rangle,|1\rangle$ are eigenstates of the number operator $a^{\dagger} a$ with corresponding eigenvalues 0 and 1 respectively, so the prediction could in principle be tested with photonic GHZ states produced in the laboratory. While a value of $N=10$ is not impossible, it is a large number of correlated modes for a practical experiment which involves multiple down-conversions and beam-splitters.

This type of symmetric GHZ state is obtainable from the usual GHZ polarized state via a local unitary transformation at each location. For example, the symmetric state given above can be prepared from an $N / 2$-photon polarization GHZ state, of a type that is found in some current experiments [28 30$]$

$$
|G H Z P\rangle=\frac{1}{\sqrt{2}}\left(|H\rangle^{\otimes N / 2}+|V\rangle^{\otimes N / 2}\right) .
$$

A possible procedure is shown in Fig. 11, where $|H\rangle,|V\rangle$ represent horizontally or vertically polarized singlephoton states, by passing each photon through a polarizing beam splitter (PBS). This converts polarization modes into spatial modes, so that $|H\rangle \equiv|1\rangle_{H}|0\rangle_{V}$ and $|V\rangle \equiv|0\rangle_{H}|1\rangle_{V}$ where $|0\rangle_{H / V}$ and $|1\rangle_{H / V}$ are number states for the horizontally and vertically polarised modes respectively. The $|G H Z P\rangle$ state then becomes $\left|\frac{N}{2}\right\rangle$ of (3.2).

On-demand state generation

Some caution is needed here in interpreting current GHZ experiments [28]. These typically involve conditional measurements, rather than a known initial quantum state. To our knowledge, current experiments only generate GHZ states in the sense that state is identified after some number of photon-counting events have already taken place. Here we assume a more traditional quantum mechanical state preparation, in that we assume a GHZ state can be generated on demand. We note that a proposal already exists for carrying out ondemand generation of a singlet state 31], which is the simplest case. Current experimental techniques for GHZ states would therefore need similar modifications to produce a well-defined GHZ state 'on demand'.

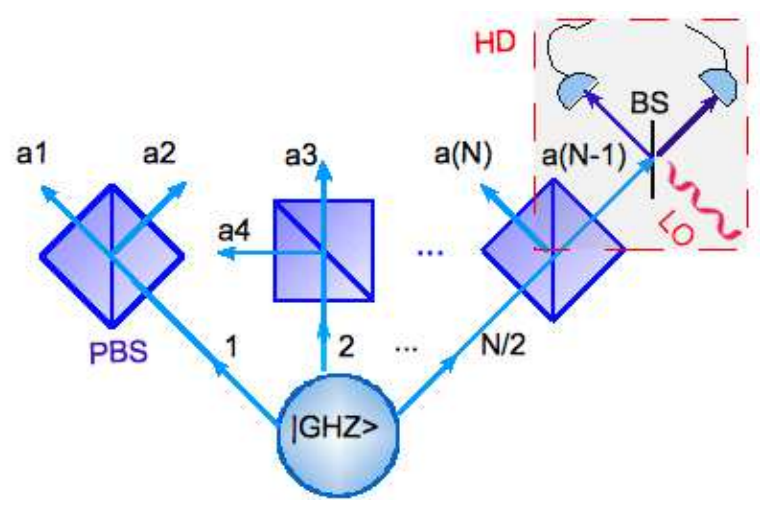

Figure 1: (Color online) Schematic of an experimental setup to test the CFRD inequalities. We assume an $N / 2$-photon GHZ state can be generated. Then by using a PBS, each photon is sent to one of the two output spatial modes depending on its polarisation. The entanglement in polarization is therefore translated into entanglement in spatial modes to produce the symmetric states $|N / 2\rangle$ with $N$ field modes. At each site, high-efficiency homodyne detection is used to measure the correlations.

\section{B. Generalized GHZ states}

A class of states that violate the CFRD inequalities is represented by the entangled states

$$
|\psi\rangle=c_{1}|0\rangle^{\otimes r}|1\rangle^{\otimes(N-r)}+c_{2}|1\rangle^{\otimes r}|0\rangle^{\otimes(N-r)} .
$$

Thus $r=N$ corresponds to extreme photon-number correlated states, a superposition of a state with 0 photons at all sites and a state with 1 photon at each site, while other values of $r$ correspond to intermediate cases. These can of course be transformed into each other with local unitary operations, although in practical terms such transformations are not always feasible without losses.

Within quantum mechanics, the $L H S$ of the CFRD inequality (3.1) is non-zero with the choice

$$
L H S=\left|\left\langle\hat{a}_{1}^{\dagger} . . \hat{a}_{r}^{\dagger} \hat{a}_{r+1} \ldots \hat{a}_{N}\right\rangle\right|^{2}=\left|c_{1} c_{2}\right|^{2}
$$

Here, $\left|c_{1}\right|^{2}+\left|c_{2}\right|^{2}=1$, and the violation is maximized for $c_{1}=c_{2}=1 / \sqrt{2}$. For the right hand side $(R H S)$ of the 
inequality, we obtain

$$
\begin{aligned}
R H S & =\left\langle\left(\hat{a}_{1}^{\dagger} \hat{a}_{1}+1 / 2\right) \ldots\left(\hat{a}_{N}^{\dagger} \hat{a}_{N}+1 / 2\right)\right\rangle \\
& =c_{1}^{2}(1 / 2)^{r}(3 / 2)^{N-r}+c_{2}^{2}(1 / 2)^{N-r}(3 / 2)^{r}(3.6)
\end{aligned}
$$

If we use photon-number correlated states with $r=N / 2$,

$$
R H S=\left(\frac{3}{4}\right)^{N / 2},
$$

which is independent of the amplitudes $c_{1}, c_{2}$.

Alternately, if we fix $c_{1}=c_{2}=1 / \sqrt{2}$ but change $r$,

$$
R H S=\frac{3^{N-r}+3^{r}}{2^{N+1}} .
$$

There is a violation of the inequality when the Bell observable $B_{N}=L H S / R H S>1$. We note from Fig. 2 that no violations are possible for $r=N$, and the optimal case has $r$ approximately equal to $N / 2$. For $N$ even, where $r=N / 2$ there is a violation if

$$
B_{N}=\frac{1}{4}\left(\frac{4}{3}\right)^{\frac{N}{2}}>1
$$

in agreement with Cavalcanti et al. [18] who have shown this requires $N \geq 10$. For odd $N$, we select $r=(N \pm 1) / 2$ to obtain a violation if

$$
2^{N-1}>3^{(N-1) / 2}+3^{(N+1) / 2}
$$

which is obtained for $N \geq 11$.

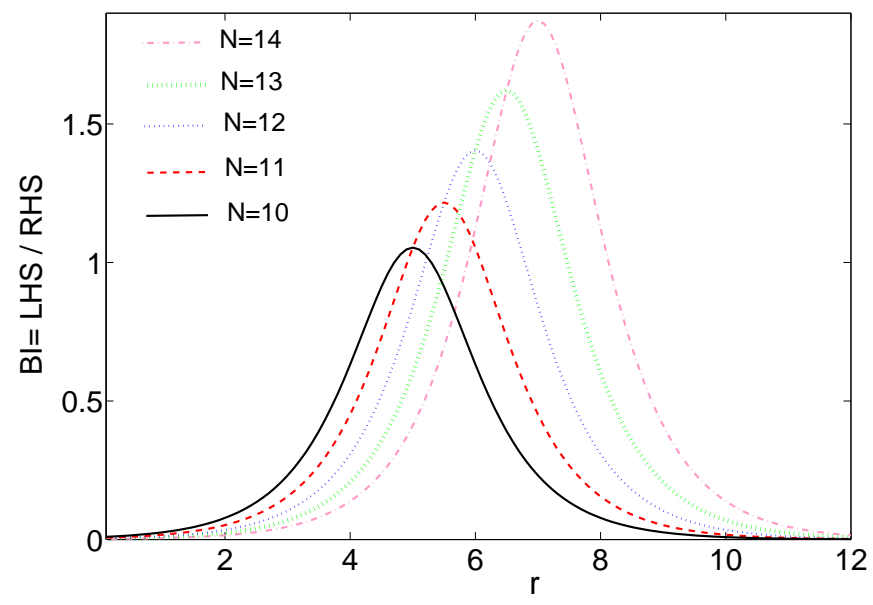

Figure 2: (Color online) Bell violation as a function of $r$ with $c_{1}=c_{2}=1 / \sqrt{2}$. Different $N(10-14)$ values are distinguished by their line style. The allowed values of $r$ are of course only the natural numbers; the lines are drawn to guide the eye.

\section{Cluster and W states}

Other forms of multipartite entangled states, such as W-states and cluster states [26, 27], are also of interest, either because they are simpler to generate experimentally, or are more resistant to certain forms of decoherence. In addition to the GHZ states [28], four and sixphoton cluster states have been realized experimentally [29, 30]. We thus present initial results for the CFRD inequality using cluster and W-states.

Consider first the four-qubit cluster state [27, 29, 30] defined as

$$
\begin{aligned}
|C\rangle= & \frac{1}{2}(|1\rangle|1\rangle|1\rangle|1\rangle+|1\rangle|1\rangle|0\rangle|0\rangle \\
& +|1\rangle|1\rangle|0\rangle|0\rangle-|0\rangle|0\rangle|0\rangle|0\rangle)
\end{aligned}
$$

and also consider the four-qubit W-state [26]

$$
\begin{aligned}
|W\rangle= & \frac{1}{2}(|1\rangle|0\rangle|0\rangle|0\rangle+|0\rangle|1\rangle|0\rangle|0\rangle \\
& +|0\rangle|0\rangle|1\rangle|0\rangle+|0\rangle|0\rangle|0\rangle|1\rangle) .
\end{aligned}
$$

In this case the qubit values correspond to the occupation number ( 0 or 1 ) of a spatial mode, as described in Sections IIIA and IIIB. The cluster state of (3.11) is a superposition of two terms which correspond to GHZ states of type (3.4) with $r=N / 2$ and $r=N$ respectively. From Fig. 2] we note there is no Bell violation when $r=N$. The effect is that this cluster state case is less optimal than the symmetric GHZ state $(r=N / 2)$ and no violation of the $N=4 \mathrm{CFRD}$ inequality is observed. For W-states (3.12), the value of the $L H S$ of (3.5) is always 0 so that no violations of this particular CFRD inequality are found. Similarly negative results were obtained for the arbitrary $N$ extensions of these superpositions $|\psi\rangle=$ $\left(|1\rangle^{\otimes N}+|0\rangle^{\otimes N / 2}|1\rangle^{\otimes N / 2}+|1\rangle^{\otimes N / 2}|0\rangle^{\otimes N / 2}-|0\rangle^{\otimes N}\right) / 2$, where $N$ was increased.

We can also consider the specific four-photon cluster state that has been produced experimentally by $\mathrm{P}$. Walther et al 29]:

$$
\begin{aligned}
|C\rangle= & \frac{1}{2}(|H\rangle|H\rangle|H\rangle|H\rangle+|H\rangle|H\rangle|V\rangle|V\rangle \\
& +|V\rangle|V\rangle|H\rangle|H\rangle-|V\rangle|V\rangle|V\rangle|V\rangle)
\end{aligned}
$$

where $|H\rangle$ and $|V\rangle$ represent respectively horizontally and vertically polarized single-photon states. Thus, $|H\rangle \equiv|1\rangle_{H}|0\rangle_{V}$ and $|V\rangle \equiv|0\rangle_{H}|1\rangle_{V}$, the $|0\rangle_{H / V}$ and $|1\rangle_{H / V}$ being number states for each polarized mode denoted by subscript $H$ and $V$. The state (3.17) is a four photon state and the qubit value is encoded in the polarisation. We also consider the four-photon W-state:

$$
\begin{aligned}
|W\rangle= & \frac{1}{2}(|V\rangle|H\rangle|H\rangle|H\rangle+|H\rangle|V\rangle|H\rangle|H\rangle \\
& +|H\rangle|H\rangle|V\rangle|H\rangle+|H\rangle|H\rangle|H\rangle|V\rangle) .
\end{aligned}
$$

As explained in Section 3A, these states can be converted to the states $\left|\frac{N}{2}\right\rangle$ of $(3.2)$ with $N=8$ via the apparatus 
of Fig. 1. We can then use the $\mathrm{N}=8 \mathrm{CFRD}$ inequality for testing the nonlocal behaviour of these states, which are written explicitly as

$$
\begin{aligned}
|C\rangle= & \frac{1}{2}(|1\rangle|0\rangle|1\rangle|0\rangle|1\rangle|0\rangle|1\rangle|0\rangle+|1\rangle|0\rangle|1\rangle|0\rangle|0\rangle|1\rangle|0\rangle|1\rangle \\
& +|0\rangle|1\rangle|0\rangle|1\rangle|1\rangle|0\rangle|1\rangle|0\rangle \\
& -|0\rangle|1\rangle|0\rangle|1\rangle|0\rangle|1\rangle|0\rangle|1\rangle)
\end{aligned}
$$

and

$$
\begin{aligned}
|W\rangle= & \frac{1}{2}(|0\rangle|1\rangle|1\rangle|0\rangle|1\rangle|0\rangle|1\rangle|0\rangle+|1\rangle|0\rangle|0\rangle|1\rangle|1\rangle|0\rangle|1\rangle|0\rangle \\
& +|1\rangle|0\rangle|1\rangle|0\rangle|0\rangle|1\rangle|1\rangle|0\rangle \\
& +|1\rangle|0\rangle|1\rangle|0\rangle|1\rangle|0\rangle|0\rangle|1\rangle) .
\end{aligned}
$$

Evaluation of the left- and right- hand sides of the CFRD Bell inequality (3.1) gives: $L H S=1 / 16$ and $R H S=$ $3^{2} / 2^{8}$. Thus, no violations are possible for the $N=8$ CFRD inequality with these 4 photon states so that in this case the cluster state is less optimal than the $r=N / 2$ GHZ state (3.4). However if we consider the $N$ photon extension of the 4-photon states (3.17) and (3.15):

$$
\begin{aligned}
|C\rangle= & \frac{1}{2}\left(|H\rangle^{\otimes N}+|H\rangle^{\otimes N / 2}|V\rangle^{\otimes N / 2}\right. \\
& \left.+|V\rangle^{\otimes N / 2}|H\rangle^{\otimes N / 2}-|V\rangle^{\otimes N}\right),
\end{aligned}
$$

we find a more positive result: $L H S=1 / 16$ and $R H S=$ $(3 / 4)^{N / 2}$, so that a violation is possible when $N \geq 20$. We note in this case no violations are obtained for the $W$ states.

\section{Angular dependence}

The CFRD inequality holds for arbitrary measurements and is therefore not restricted to the orthogonal choice specified in the previous section. Noting that Bell inequalities for correlated spins are optimized at polarizer angles that are distinct at different sites, we wish to investigate the effect of an arbitrary local oscillator phase.

Using general quadrature operators $\hat{X}_{n}^{\theta}=\left(\hat{a}_{n} e^{-i \theta_{n}}+\right.$ $\left.\hat{a}_{n}^{\dagger} e^{i \theta_{n}}\right) / 2$ for each site $n$, and defining $\hat{F}_{n}=\hat{X}_{n}^{\theta_{n}}+i \hat{X}_{n}^{\theta_{n}^{\prime}}$, we substitute these in the CFRD inequality (2.1) with $x_{n}=\hat{X}_{n}^{\theta}$ and $p_{n}=\hat{X}_{n}^{\theta^{\prime}}$. The inequality then becomes

$$
\begin{aligned}
& \left|\left\langle\prod_{n=1}^{N} F_{n}\right\rangle_{P}\right|^{2} \leq\left\langle\prod _ { n = 1 } ^ { N } \left[ a_{n}^{\dagger} a_{n}\right.\right. \\
& \left.\left.\quad+\frac{1}{4} a_{n}^{2}\left(e^{-2 i \theta_{n}}+e^{-2 i \theta_{n}^{\prime}}\right)+\frac{1}{4} a_{n}^{\dagger 2}\left(e^{2 i \theta_{n}}+e^{2 i \theta_{n}^{\prime}}\right)\right]\right\rangle_{P},
\end{aligned}
$$

which gives additional terms in the RHS. We note that in QM, these extra terms correspond to operators that change the boson number by two. Hence for states like $|0011\rangle+|1100\rangle$ they will be zero, leaving the RHS invariant with the choice of phases $\theta_{n}, \theta_{n}^{\prime}$. Therefore, for GHZ states, the RHS will be just as in Eq (3.6).

For simplicity, we treat the case of $N=2$ as an illustration. Within QM, the $L H S$ for the Bell state $|\psi\rangle=c_{1}|0\rangle|1\rangle+c_{2}|1\rangle|0\rangle$ is:

$$
\begin{aligned}
L H S= & \mid\left\langle\left.\left(\hat{X}_{1}^{\theta}+i \hat{X}_{1}^{\theta^{\prime}}\right)\left(\hat{X}_{2}^{\phi}+i \hat{X}_{2}^{\phi^{\prime}}\right)\right|^{2}\right. \\
= & \mid\left\langle\left[\left(\hat{x}_{1} \cos \theta+\hat{p}_{1} \sin \theta\right)+i\left(\hat{x}_{1} \cos \theta^{\prime}+\hat{p}_{1} \sin \theta^{\prime}\right)\right]\right. \\
\times & {\left.\left[\left(\hat{x}_{2} \cos \phi+\hat{p}_{2} \sin \phi\right)+i\left(\hat{x}_{2} \cos \phi^{\prime}+\hat{p}_{2} \sin \phi^{\prime}\right)\right]\right\rangle\left.\right|^{2} } \\
= & \mid \frac{1}{2} c_{1} c_{2}\left[\left(\cos (\theta-\phi)-\cos \left(\theta^{\prime}-\phi^{\prime}\right)\right)\right. \\
& \left.+i\left(\cos \left(\theta^{\prime}-\phi\right)+\cos \left(\theta-\phi^{\prime}\right)\right)\right]\left.\right|^{2}
\end{aligned}
$$

since here $\left\langle\hat{x}_{1} \hat{x}_{2}\right\rangle=\left\langle\hat{p}_{1} \hat{p}_{2}\right\rangle=c_{1} c_{2} / 2$ and $\left\langle\hat{x}_{1} \hat{p}_{2}\right\rangle=$ $\left\langle\hat{p}_{2} \hat{x}_{1}\right\rangle=0$. The LHS is maximised either with the orthogonal choice $\theta=\phi=0, \theta^{\prime}=-\pi / 2=-\phi^{\prime}$, for which $\left\langle\operatorname{Im}\left\{\hat{F}_{1} \hat{F}_{2}\right\}\right\rangle=0,\left\langle\operatorname{Re}\left\{\hat{F}_{1} \hat{F}_{2}\right\}\right\rangle=c_{1} c_{2}$; or with the choice $\theta=0, \theta^{\prime}=\pi / 2, \phi=\pi / 4, \phi^{\prime}=-\pi / 4$ for which $\left\langle\operatorname{Im}\left\{\hat{F}_{1} \hat{F}_{2}\right\}\right\rangle=c_{1} c_{2} / \sqrt{2},\left\langle\operatorname{Re}\left\{\hat{F}_{1} \hat{F}_{2}\right\}\right\rangle=c_{1} c_{2} / \sqrt{2}$. In both cases, the $L H S$ is equal to $\left|c_{1} c_{2}\right|^{2}$, as in Eq (3.5). Hence, just as in the orthogonal quadrature case, there is no violation.

The case for the extreme photon-number correlated state $|\psi\rangle=c_{1}|0\rangle|0\rangle+c_{2}|1\rangle|1\rangle$ is even less favourable. The value of $L H S$ can still be maximized to $\left|c_{1} c_{2}\right|^{2}$, with angle choice $\theta=\phi=0, \theta^{\prime}=\phi^{\prime}= \pm \pi / 2$ or $\theta=0$, $\theta^{\prime}=\pi / 2, \phi=-\pi / 4, \phi^{\prime}=\pi / 4$. However, the value of the $R H S$ is increased even further, so that again, there is no violation.

More generally, the inequality is violated for $N \geq 10$, that is, with more correlated modes and observers. We find that the optimal violations are found with phase choices: $\theta_{1}=\cdots=\theta_{n}=0$, and $\theta_{1}^{\prime}=\cdots=\theta_{r}^{\prime}=-\theta_{r+1}^{\prime}=$ $\cdots=-\theta_{n}^{\prime}=-\pi / 2$ or $\theta_{n}=(-1)^{n+1} \pi(n-1) /(2 N), \theta_{n}^{\prime}=$ $\theta_{n}+\pi / 2(n \leq r)$ and $\theta_{n}=(-1)^{n} \pi(n-1) /(2 N), \theta_{n}^{\prime}=$ $\theta_{n}-\pi / 2(n>r)$. The LHS and RHS then become, for arbitrary $N$ and $r$, with $c_{1}=c_{2}=1 / \sqrt{2}$ :

$$
\begin{aligned}
& L H S_{\text {max }}=\left|c_{1} c_{2}\right|^{2}=1 / 4, \\
& R H S_{\text {max }}=\frac{3^{N-r}+3^{r}}{2^{N+1}} .
\end{aligned}
$$

In summary, we find that the violations are independent of the relative phases between different sites [22]unlike the usual case with Bell inequalities for correlated spins-, thus confirming a result pointed out in [18] for this larger range of states.

\section{MABK INEQUALITIES}

In early proposals for obtaining inequalities to test local realism with continuous variable measurements, only the sign of the quadrature variable is recorded [8]. As far 
as LHV theories are concerned, it is just the final outcomes that are relevant, not the quantum operators corresponding to them. This allows one to use the same logic that is employed for dichotomic spin variables. Our results here are therefore similar to those of earlier workers [8, 14, 15]. As in the work of [15], we find an exponential improvement in Bell violation with increasing number of sites, but we demonstrate that result for a larger class of states, some of which may be within experimental feasibility.

\section{A. MABK approach with binned variables}

Violations of the MABK inequalities, which were originally used for spin variables, can also be obtained by using binned outcomes of continuous variable observables. These are applicable to any quantum state. Here the binned measurement $x_{n}^{\text {bin }}=2 \Theta\left(x_{n}\right)-1$ is the observable with outcome $x_{n}^{b i n}=+1$, if $x_{n} \geq 0$, and $x_{n}^{\text {bin }}=-1$, if $x_{n}<0$. We define $p_{n}^{\text {bin }}$ similarly, together with corresponding quantum operators $\hat{x}_{n}^{\text {bin }}=2 \Theta\left(\hat{x}_{n}\right)-1$ and $\hat{p}_{n}^{\text {bin }}=2 \Theta\left(\hat{p}_{n}\right)-1$. Next, we can introduce a new binned complex observable $a_{n}^{\text {bin }}$ at location $n$ through its real and imaginary parts, so that $\operatorname{Re}\left\{a_{n}^{b i n}\right\} \equiv x_{n}^{b i n}$ and $\operatorname{Im}\left\{a_{n}^{b i n}\right\}=p_{n}^{b i n}$. There is also a corresponding operator, $\hat{a}_{n}^{b i n}=\hat{x}_{n}^{b i n}+i \hat{p}_{n}^{b i n}$.

For $N$ sites, we can modify the CFRD proof directly, assuming local hidden variables, to get:

$$
\left|\left\langle a_{1}^{b i n} a_{2}^{b i n} \ldots\right\rangle_{P}\right|^{2} \leq\left\langle\prod_{n=1}^{N}\left(\left[x_{n}^{b i n}\right]^{2}+\left[p_{n}^{b i n}\right]^{2}\right)\right\rangle_{P}=2^{N}
$$

This follows because $\left\langle\left[x_{n}^{b i n}\right]^{2}\right\rangle_{P},\left\langle\left[p_{n}^{\text {bin }}\right]^{2}\right\rangle_{P}=1$. The inequality remains valid if any of the complex variables $a_{n}^{b i n}$ are replaced by their conjugates.

The CFRD inequality for this binned case then reduces to that of Mermin and Ardehali [16] by taking the square root of both of sides. However, the inequality was later strengthened by Belinskii and Klyshko[17], to give an approach which we refer to as the MABK inequality. This approach can be extended to account for more general angles and states, by introducing binned angular observables $X_{n}^{\theta, b i n}=2 \Theta\left(X_{n}^{\theta}\right)-1$. Next, $a_{n}^{b i n}$ is replaced with $F_{n}^{b i n}=X_{n}^{\theta, b i n}+i X_{n}^{\theta^{\prime}, \text { bin }}$. The notation $\theta_{n}$ and $\theta_{n}^{\prime}$ represents the angles defining the two local oscillator phases chosen at site $n$, where $k=1, \ldots, N$, giving $2^{N}$ distinct sets of measurement settings.

Then, defining $\Pi_{N}=F_{1}^{b i n} F_{2}^{b i n} \ldots F_{N}^{b i n}$, we can test for violations of the even stronger MABK inequality

$$
\left|S_{N}\right| \leq 1
$$

where

$$
S_{N}=2^{-N / 2}\left\langle\operatorname{Re}\left\{\Pi_{N}\right\} \pm \operatorname{Im}\left\{\Pi_{N}\right\}\right\rangle_{P}
$$

, for $N$ even, and

$$
S_{N}=2^{-(N-1) / 2} \operatorname{Re}(\operatorname{Im})\left\{\left\langle\Pi_{N}\right\rangle_{P}\right\}
$$

for $N$ odd. The inequality also holds for $S_{N}=$ $2^{-(N-1) / 2}\left\langle\sqrt{\left(\operatorname{Re}\left\{\Pi_{N}\right\}\right)^{2}+\left(\operatorname{Im}\left\{\Pi_{N}\right\}\right)^{2}}\right\rangle_{P}$. These inequalities have been considered recently for the case of GHZ states (2.3), where $r=N$, by Acin et al [15]. We can also define $\Pi_{N}$ with the adjoint operators at any of the sites, to obtain the same inequality.

\section{B. Three-site example}

Within quantum mechanics, the $L H S$ of the Bell inequality (4.2) can be calculated via binned quadrature phase amplitudes. It is instructive to give an example of the calculation in detail. For the state (2.3), the lowest values of $N$ and $r$ that give a violation are $N=3$ and $r=1$ :

$$
|\psi\rangle=\frac{1}{\sqrt{2}}\left(|0\rangle_{1}|1\rangle_{2}|1\rangle_{3}+|1\rangle_{1}|0\rangle_{2}|0\rangle_{3}\right) .
$$

Using the harmonic oscillator wavefunctions given in the Appendix, we find that:

$$
\begin{aligned}
\left\langle\hat{\Pi}_{3}\right\rangle= & \left\langle\hat{F}_{1}^{b i n} \hat{F}_{2}^{b i n} \hat{F}_{3}^{b i n}\right\rangle \\
= & \left(\frac{2}{\pi}\right)^{3 / 2}\left[\cos (\theta-\phi-\gamma)-\cos \left(\theta-\phi^{\prime}-\gamma^{\prime}\right)\right. \\
& -\cos \left(\theta^{\prime}-\phi^{\prime}-\gamma\right)-\cos \left(\theta^{\prime}-\phi-\gamma^{\prime}\right) \\
& +i\left(\cos \left(\theta-\phi^{\prime}-\gamma\right)+\cos \left(\theta-\phi-\gamma^{\prime}\right)\right. \\
& \left.\left.+\cos \left(\theta^{\prime}-\phi-\gamma\right)-\cos \left(\theta^{\prime}-\phi^{\prime}-\gamma^{\prime}\right)\right)\right]
\end{aligned}
$$

where we denote $\theta_{1}=\theta, \theta_{2}=\phi$, and $\theta_{3}=\gamma$.

Choosing the two measurements made at each site to be orthogonal, and measurements at all sites along the same (or opposite) directions, so that $\theta=\phi=\gamma=0$, and $\theta^{\prime}=-\phi^{\prime}=-\gamma^{\prime}=-\pi / 2$ (which means that $\Pi_{3}=$ $\left.a_{b i n}^{\dagger} b_{b i n} c_{b i n}\right)$, we obtain

$$
S_{3}=2^{-(3-1) / 2} \sqrt{\left(\operatorname{Re}\left\{\Pi_{3}\right\}\right)^{2}+0}=2(2 / \pi)^{3 / 2}>1 .
$$

Alternatively, using different measurement angles: $\theta=$ $0, \phi=-\pi / 6, \gamma=-2 \pi / 6, \theta^{\prime}=\pi / 2, \phi^{\prime}=-4 \pi / 6, \gamma^{\prime}=$ $-5 \pi / 6$, we can rotate the $L H S$ moment, so that

$$
S_{3}=2^{-(3-1) / 2} \sqrt{0+\left(\operatorname{Im}\left\{\Pi_{3}\right\}\right)^{2}}=2(2 / \pi)^{3 / 2}>1 .
$$

Next, consider the extreme GHZ state of form (2.3) with $r=3$ :

$$
|\psi\rangle=\frac{1}{\sqrt{2}}\left(|0\rangle_{1}|0\rangle_{2}|0\rangle_{3}+|1\rangle_{1}|1\rangle_{2}|1\rangle_{3}\right) .
$$

We obtain a new $\left\langle\Pi_{3}\right\rangle$ with $\cos (\theta+\phi+\gamma), \cos \left(\theta+\phi^{\prime}+\gamma^{\prime}\right)$, etc. replacing $\cos (\theta-\phi-\gamma), \cos \left(\theta-\phi^{\prime}-\gamma^{\prime}\right)$, etc. in 
Eq. (4.6). In this case the choice $\theta=0, \phi=\pi / 6, \gamma=$ $2 \pi / 6, \theta^{\prime}=\pi / 2, \phi^{\prime}=4 \pi / 6, \gamma^{\prime}=5 \pi / 6$, gives the maximum violation of the inequality,

$$
S_{3}=2^{-(3-1) / 2} \sqrt{\left(\operatorname{Re}\left\{\Pi_{3}\right\}\right)^{2}+0}=2(2 / \pi)^{3 / 2}>1,
$$

as has been presented by Acin et al.

\section{N-site inequalities}

Continuing in this fashion for higher $N$, we find that

$$
S_{N}=\frac{\sqrt{2}}{2}\left(\frac{4}{\pi}\right)^{N / 2}
$$

for the choice $\theta_{n}=(-1)^{N+1} \pi(k-1) /(2 N), \theta_{n}^{\prime}=\theta_{n}+$ $\pi / 2$ for $k \leq r$, and $\theta_{n}=(-1)^{N} \pi(n-1) /(2 N), \theta_{n}^{\prime}=$ $\theta_{n}-\pi / 2$ for $n>r$. There is a violation of the Bell inequality when $S_{N}>1$. This result has been presented by Acin et al. for the case of $r=N$. We confirm the exponential increase with number of sites $N$, but also make the observation that the violation occurs for all types of states of the form (2.3), independently of $r$. This contrasts with the result for the CFRD inequality, which is strongly dependent on $r$, requiring $r \sim N / 2$. Violation of the BI with binning is therefore possible in principle for $N \geq 3$, but, as shown in later sections, this strategy is very sensitive to losses and noise.

\section{Cluster and W states}

We also present initial results for the violations of the MABK CV inequalities using the specific cluster and Wstates (3.11) and (3.12) We first consider the case $N=$ 4 with $\mathrm{CV}$ binned outcomes. Using the same method as above, and selecting the optimal choice of angles, we obtain the best $\mathrm{N}=4 \mathrm{MABK}$ correlations of

$$
\begin{aligned}
& \left|S_{4-C}\right|=\frac{\sqrt{2}}{4}\left(\frac{4}{\pi}\right)^{2}<\left|S_{4-G H Z}\right|=\frac{\sqrt{2}}{2}\left(\frac{4}{\pi}\right)^{2} \\
& \left|S_{4-W}\right|=0<\left|S_{4-G H Z}\right|
\end{aligned}
$$

for cluster and W-states respectively, and thus we see no violation here for $N=4$. However, on examining the generalisation

$|\psi\rangle=\left(|1\rangle^{\otimes N}+|0\rangle^{\otimes N / 2}|1\rangle^{\otimes N / 2}+|1\rangle^{\otimes N / 2}|0\rangle^{\otimes N / 2}-|0\rangle^{\otimes N / 2}\right) / 2$

of the superpositions (3.11) and (3.12) for higher $N$, we find $\left|S_{N-C}\right|=\frac{\sqrt{2}}{4}\left(\frac{4}{\pi}\right)^{N / 2}$ so that violations are possible for $N \geq 10$. Calculation for the $\mathrm{W}$-states shows no violation for any $N$. These calculations are specific to the case where the qubit is realized as a photon number of 1 or 0 at a given spatial mode. The cases (3.17) - (3.16) where the qubit is realized in terms of horizontal or vertical polarisation, as explained in Section III, could also be examined.We leave open the question of whether other Bell inequalities might be more sensitive for the cluster and $\mathrm{W}$-states with continuous variables measurements, as shown by Scarani et al [32] for spin measurements.

\section{FUNCTIONAL OPTIMIZATION}

In this section, we show that greatly improved experimental simplicity, together with robustness against decoherence is possible by testing local realism using a functional moment inequality approach. The advantage of the CFRD moment approach compared to the binning approach [8] is that it gives a low weight to quadrature signals with low amplitude, which reduces the sensitivity to noise. On the other hand, binning has the advantage that it saturates, which increases the relative size of the violations. We show that an approach that combines these two features using functions of quadrature variables is possible.

We analytically calculate the optimal function using a variational calculus method. This produces an inequality which is violated by the states of Eq. (3.4) for $N \geq 5$, i.e. half the number of modes required with the method of [18]. The violation increases exponentially with $N$, and we will see that the critical detection efficiency $\eta_{\text {crit }}$ decreases asymptotically to 0.69 , a significant reduction.

\section{A. The functional inequalities}

Functional inequalities are already implicit in the original derivation of CFRD [18]. For completeness, we present a proof that takes explicit account of functions of measurements that can be made at each of $N$ causally separated sites. We consider the measurable joint probability $P\left(X_{1}^{\theta}, X_{2}^{\phi} \ldots\right)$ for outcome $X_{1}^{\theta}, X_{2}^{\phi}, \ldots$ at locations $1,2, \ldots$, respectively, where $\theta, \phi, \ldots$ represents a choice of measurement parameter. For local hidden variable (LHV) theories, the joint probability is written [1] in terms of variables $\lambda$, as

$$
P\left(X_{1}^{\theta}, X_{2}^{\phi}, \ldots\right)=\int_{\lambda} d \lambda P(\lambda) P\left(X_{1}^{\theta} \mid \lambda\right) P\left(X_{2}^{\phi} \mid \lambda\right) \ldots
$$

where $P\left(X_{1}^{\theta} \mid \lambda\right)$ is the probability for result $X_{1}^{\theta}$ given that the system is specified by the hidden variable state $\lambda$. We note that while we use the term "hidden variable state", the states can in fact be quantum states, denoted $\hat{\rho}_{\lambda}$, as long as they are separable: i.e., not entangled. The assumption of local causality (or separability) allows the factorization in the integrand. From this, it also follows that we can write the measurable moments $\left\langle X_{1} X_{2} X_{3} \ldots\right\rangle$, where $X_{n}$ is an observable associated with a measurement at site $n$, etc., as

$$
\left\langle X_{1}^{\theta} X_{2}^{\phi} X_{3}^{\gamma} \ldots\right\rangle_{P}=\int_{\lambda} d \lambda P(\lambda)\left\langle X_{1}^{\theta}\right\rangle_{\lambda}\left\langle X_{2}^{\phi}\right\rangle_{\lambda}\left\langle X_{3}^{\gamma}\right\rangle_{\lambda} \ldots
$$


Here $\left\langle X_{n}^{\theta}\right\rangle_{\lambda}$ is the average of $X_{n}^{\theta}$ given the local hidden variable state $\lambda$, which means that

$$
\left\langle X_{n}^{\theta}\right\rangle_{\lambda}=\int d X_{n}^{\theta} P\left(X_{n}^{\theta} \mid \lambda\right) X_{n}^{\theta},
$$

where $X_{n}^{\theta}$ denotes the outcome of measurement at site $n$ with phase $\theta$.

Next we construct, for each site $n$, real functions of the two observables $f_{n}\left(X_{n}^{\theta}\right), g_{n}\left(X_{n}^{\theta^{\prime}}\right)$, and define the complex function:

$$
F_{n}=f_{n}\left(X_{n}^{\theta}\right)+i g_{n}\left(X_{n}^{\theta^{\prime}}\right) .
$$

The complex moment $\left\langle F_{1} F_{2} \ldots F_{N}\right\rangle$ can be expressed using sums of real-valued expressions of the type $\left\langle f_{1}\left(X_{1}^{\theta}\right) g_{2}\left(X_{2}^{\phi^{\prime}}\right) \ldots f_{N}\left(X_{N}^{\varphi}\right)\right\rangle$. Of course, $f_{n}\left(X_{n}^{\theta}\right)$ is just another observable composed of the observable $X_{n}^{\theta}$ plus some local post-measurement processing. This postprocessing can be carried out at any time subsequent to the measurement. Eq. (5.2) is therefore valid for

$$
\left\langle f\left(X_{1}^{\theta}\right) \ldots f\left(X_{N}^{\varphi}\right)\right\rangle_{P}=\int_{\lambda} d \lambda P(\lambda)\left\langle f\left(X_{1}^{\theta}\right)\right\rangle_{\lambda} \ldots\left\langle f\left(X_{N}^{\varphi}\right)\right\rangle_{\lambda} .
$$

The expectation value of products of the $F_{n}$ must satisfy:

$$
\left\langle F_{1} \ldots F_{N}\right\rangle_{P}=\int_{\lambda} d \lambda P(\lambda)\left\langle F_{1}\right\rangle_{\lambda} \ldots\left\langle F_{N}\right\rangle_{\lambda}
$$

where $\left\langle F_{n}\right\rangle_{\lambda} \equiv\left\langle f_{n}\left(X_{n}^{\theta}\right)\right\rangle_{\lambda}+i\left\langle g_{n}\left(X_{n}^{\theta^{\prime}}\right)\right\rangle_{\lambda}$. Hence, from (5.6), the following inequality holds:

$$
\left|\left\langle F_{1} F_{2} \ldots F_{N}\right\rangle_{P}\right|^{2} \leq \int d \lambda P(\lambda)\left|\left\langle F_{1}\right\rangle_{\lambda}\right|^{2} \ldots\left|\left\langle F_{N}\right\rangle_{\lambda}\right|^{2}(5.7)
$$

For any particular value of $\lambda$, the statistics predicted for $f_{n}\left(X_{n}\right)$ must have a non-negative variance, i.e., $\left\langle f_{n}\left(X_{n}\right)\right\rangle_{\lambda}^{2} \leq\left\langle f_{n}\left(X_{n}\right)^{2}\right\rangle_{\lambda}$. Then we can rewrite (5.7) explicitly in terms of the $f_{n}$ 's. Using this variance inequality we arrive at a functional moment inequality:

$$
B=\frac{\left|\left\langle\prod_{n=1}^{N}\left[f_{n}\left(X_{n}^{\theta}\right)+i g_{n}\left(X_{n}^{\theta^{\prime}}\right)\right]\right\rangle\right|^{2}}{\left\langle\prod_{n=1}^{N}\left[f_{n}\left(X_{n}^{\theta}\right)^{2}+g_{n}\left(X_{n}^{\theta^{\prime}}\right)^{2}\right]\right\rangle} \leq 1 .
$$

We will measure the violation of this inequality by the Bell observable $B$ defined above, so that failure of LHV is demonstrated when $B>1$.

\section{B. Functional inequality examples}

First, we investigate three specific types of function:

1. Fractional order moments: $f_{n}(x)=g_{n}(x)=$ $|x|^{m} \operatorname{sign}(x)$.

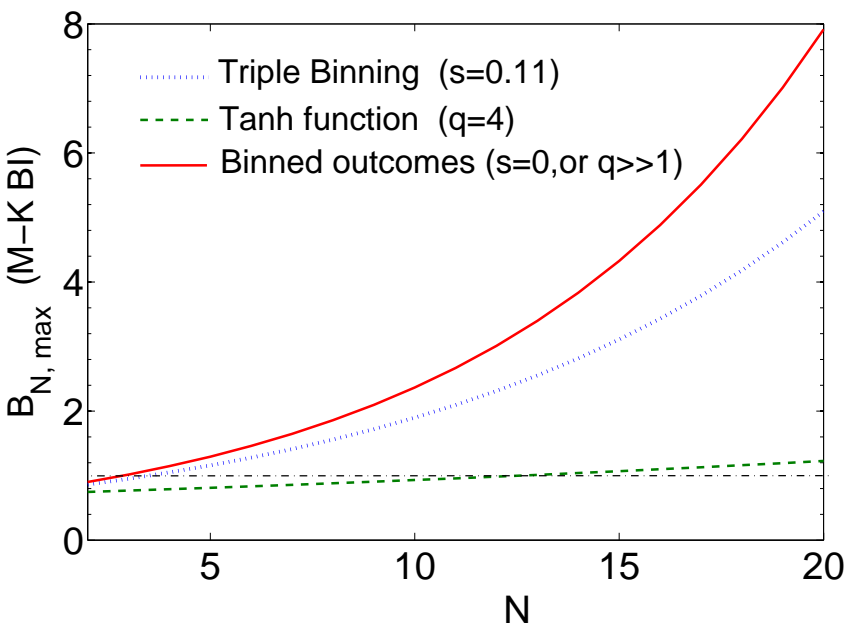

Figure 3: (Color online) Maximum violations of MABK Bell inequalities as a function of the number of modes $N$ with ideal detection efficiency. Violation of the Bell inequalities is achieved when $B>1$. The triple step function case is the dotted line with $s=0.11$, which reduces to the binned case when $s=0$ (solid line); the tanh function case is the dashed line with $q=4, m=1.4$, which reduces to the binned case when $q$ is large. Here the parameters $m, q$, and $s$ are the optimal choices to maximize the Bell value.

2. Triple binning: $f_{n}(x)$ is the triple-valued step function equal to -1 when $x<-s,+1$ when $x>s$, and 0 elsewhere.

3. Powers of tanh functions: $f_{n}(x)=g_{n}(x)=$ $\operatorname{sign}(x)|\tanh (q x)|^{m}$.

For all of these functions, we obtain an LHV violation when $N \geq 5$, with optimal choice of $m, s$, and $q$. This is a much more feasible experiment than that for an integer moment with $m=1$, which required $N \geq 10$ in CFRD. The reason for this is that these three functions all combine high intensity saturation with small-noise insensitivity. The region close to 0 is relatively flat, rather than discontinuous as in the binning approach. This leads to reduced sensitivity to small noise effects, which otherwise will lead to a greatly amplified contribution of random vacuum noise to the correlations. Here, we again select the optimal $r$ at $r=N / 2(N$ is even $)$ or at $r=(N \pm 1) / 2$ ( $N$ is odd). The extreme state where $r=N$ is less optimal.

It is worth noting that bounded CV observables (cases 2 and 3 above) can also be used with the MABK inequalities. These also reduce to the binning results asymptotically as $s=0$ in the triple binning case and as $q \rightarrow \infty$ or $m \rightarrow 0$ in the tanh case, as shown in Fig. 3. Small $q$ is less optimal as shown by dashed curve. However, for the MABK inequalities the triple binning case $(s \neq 0)$ is less optimal than the standard binned case $(s=0)$ even if we select a nonzero optimal value of $s$. 


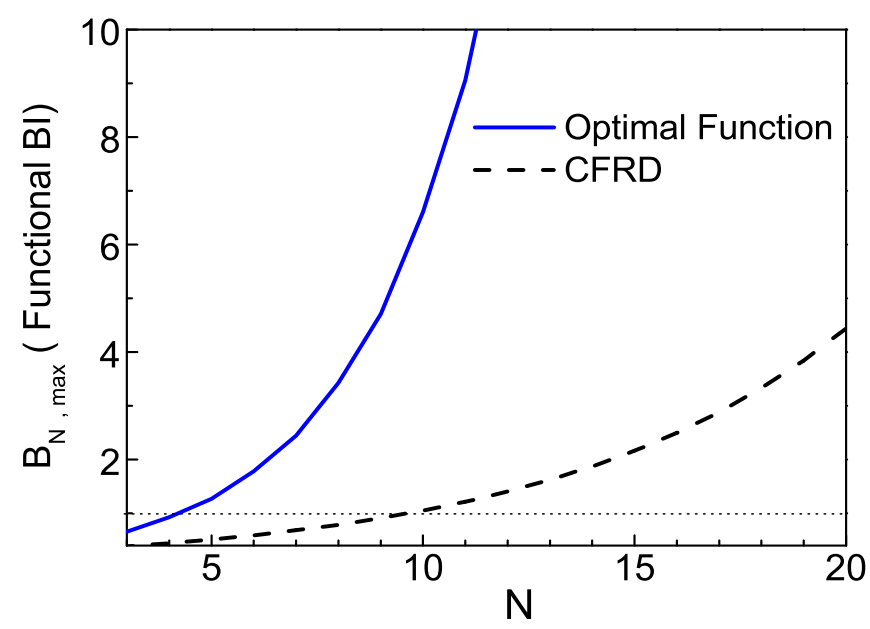

Figure 4: (Color online) Maximum violations of functional CV inequality with GHZ states as a function of the number of modes $N$. Violation of the Bell inequality is achieved when $B>1$. The violations using the optimal function (solid) are much stronger than the CFRD result (dashed).

\section{Optimized functional CFRD}

In order to get the strongest violation of LHV theories, we can optimize the function of observables by using variational calculus:

$\frac{\delta B}{\delta f_{n}}=\frac{\delta B}{\delta g_{n}}=\frac{\delta}{\delta f_{n}}\left[\frac{\left|\left\langle\prod_{i=1}^{N}\left\{f_{n}\left(\hat{X}_{n}^{\theta}\right)+i g_{n}\left(\hat{X}_{n}^{\theta^{\prime}}\right)\right\}\right\rangle\right|^{2}}{\left\langle\prod_{i=1}^{N}\left\{f_{n}\left(\hat{X}_{n}^{\theta}\right)^{2}+g_{n}\left(\hat{X}_{n}^{\theta^{\prime}}\right)^{2}\right\}\right\rangle}\right]=0$.

For simplicity, we assume the functions $f_{n}$ and $g_{n}$ are odd. The numerator can be maximized by choosing orthogonal angles, while the denominator of the fraction is invariant with angles. In the case of the GHZ states, we find that:

$$
B_{N}=\frac{2^{N-1}\left(\frac{2}{\pi}\right)^{\frac{N}{2}}\left(\prod_{n=1}^{N} I_{n}^{+}+\prod_{n=1}^{N} I_{n}^{-}\right)^{2}}{\prod_{n=1}^{r} I_{n} \prod_{k=r+1}^{N} I_{n}^{o}+\prod_{n=1}^{r} I_{n}^{o} \prod_{k=r+1}^{N} I_{n}}
$$

where $I_{n}^{ \pm}=2 \int e^{-2 x^{2}} x f_{n}^{ \pm} d x, I_{n}=4 \int x^{2} e^{-2 x^{2}}\left[\left(f_{n}^{+}\right)^{2}+\right.$ $\left.\left.\left(f_{n}^{-}\right)^{2}\right)\right] d x$, and $\left.I_{n}^{o}=\int e^{-2 x^{2}}\left[\left(f_{n}^{+}\right)^{2}+\left(f_{n}^{-}\right)^{2}\right)\right] d x$ (given in the Appendix) are different integrals for $x$ which contribute to the expectation values in both sides of inequality (5.8). Here $f_{n}^{ \pm}=f_{n} \pm g_{n}$, and the factor $e^{-2 x^{2}}$ was obtained from the joint probability of observables. Requiring $\delta B_{N} / \delta f_{n}^{ \pm}=0$, we find the optimal condition: $f_{n}(x)= \pm g_{n}(x)$. The components of complex functions $f_{n}, g_{n}$ are the same at each site, and have the form

$$
f_{n}(x)=g_{n}(x)=\frac{x}{1+\varepsilon_{N} x^{2}} .
$$

For the even $N$ case, it is optimal to choose $r=N / 2$. Then $\varepsilon_{N}$ is independent of $N$, but has to be calculated numerically since it satisfies a nonlinear integral equation: $\varepsilon_{N}=4 I^{\circ} / I$. Using the method shown in the Appendix, we can obtain the fixed values of integrals. We find that $\varepsilon_{N}=2.9648$ gives the optimal case, and $B_{N}=2^{N-2}\left[2\left(I^{+}\right)^{4} /\left(\pi I^{\circ} I\right)\right]^{N / 2}$.

For $N$ an odd number, the greatest violations occur for $r=(N-1) / 2$. The optimal function has the same form as in (5.11) except that the parameter $\varepsilon_{N}$ changes to $\varepsilon_{N}^{\prime}$, where:

$$
\varepsilon^{\prime}{ }_{N} \equiv \varepsilon_{N}\left[\frac{N \varepsilon_{N}^{+}-\varepsilon_{N}^{-}}{N \varepsilon_{N}^{+}+\varepsilon_{N}^{-}}\right]
$$

and $\varepsilon_{N}^{ \pm}=\varepsilon_{N} \pm 4$. However, the numerical value of $\varepsilon_{N}$ and $\varepsilon_{N}^{\prime}$ now depend on $N$, as the integral equation (5.12) for odd values of $N$ is $N$-dependent. This provides better violations of (5.8) than any other arbitrary function, provided $N \geq 5$. The maximum $B_{N}$ value with this optimal choice is shown in Fig. 4 compared with the CFRD result which uses a simple correlation function.

In summary, a functional optimization approach gives a substantially larger violation of the CFRD inequality, and we have carried this optimization out explicitly for the case of a generalized GHZ state.

\section{MANY OBSERVABLES PER SITE: SV INEQUALITIES}

In this Section, we compare the CFRD inequality with the inequalities given by Shchukin and Vogel (SV) [19]. These generalize the CFRD inequality to measurements with 4 or 8 settings or observables at each site. In principle, this may allow larger violations or greater robustness against loss and noise. However, these are increasingly more complicated both to analyse and to carry out experiments for. By comparison, the CFRD approach considers just two detector settings per site.

\section{A. Four setting SV inequality}

First, we evaluate the results of SV for 4 observables per site, using arbitrary choices of phase for the quadrature measurements, where $F_{n}=\left(X_{n}^{\theta_{n}^{1}}\right)^{m}+i\left(X_{n}^{\theta_{n}^{2}}\right)^{m}+$ $j\left(X_{n}^{\theta_{n}^{3}}\right)^{m}+k\left(X_{n}^{\theta_{n}^{4}}\right)^{m}$, are quaternionic functions of observables. Here $X_{n}^{\theta_{n}^{\ell}},(\ell=1,2,3,4)$ are real observables at site $n$. The multiplication rules for the quaternionic units $i, j$, and $k$ are $i^{2}=j^{2}=k^{2}=-1$, $i j=-j i=k, j k=-k j=i, k i=-i k=j$. Thus, Shchukin and Vogel (SV) [19] obtain the following inequality within any hidden-variable theory:

$$
\left|\left\langle F_{1} F_{2} \ldots\right\rangle_{P}\right|^{2} \leq\left\langle\sum_{l=1}^{4}\left(X_{1}^{\theta_{1}^{\ell}}\right)^{2 m} \times \sum_{l=1}^{4}\left(X_{2}^{\theta_{2}^{\ell}}\right)^{2 m} \ldots\right\rangle_{P}(6.1)
$$


We consider the class of entangled states of type 3.4 to find violations of this inequality. We use the optimal parameters found for the CFRD inequality, $r=N / 2$ and $c_{1}=c_{2}=1 / \sqrt{2}$, for comparison with the $\mathrm{SV}$ inequalities with 4 observables per site.

\section{Two-site case}

As these inequalities are more complicated theoretically, we begin with the simplest example of $N=2$. At site $n=1$, we have 4 choices of observables $\left(X_{A}^{\theta_{1}^{l}}\right)^{m}(l=$ $1,2,3,4)$ with corresponding outcomes $\left|x_{A}^{\theta_{1}^{l}}\right|^{m} \operatorname{sign}\left(x_{A}^{\theta_{1}^{l}}\right)$, and similarly for the other site. The values of $L H S$ and RHS are evaluated in terms of integrals for $x$. First we evaluate the inequalities for $N=2$, with the state $|\psi\rangle=c_{1}|0\rangle|1\rangle+c_{2}|1\rangle|0\rangle$, using $c_{1}=c_{2}=1 / \sqrt{2}$ and $m=1 / 3$. The LHS of the SV inequality has many terms:

$$
\begin{aligned}
L H S= & \mid\left\langle\left[\left(\hat{X}_{A}^{\theta^{1}}\right)^{\frac{1}{3}}+i\left(\hat{X}_{A}^{\theta^{2}}\right)^{\frac{1}{3}}+j\left(\hat{X}_{A}^{\theta^{3}}\right)^{\frac{1}{3}}+k\left(\hat{X}_{A}^{\theta^{4}}\right)^{\frac{1}{3}}\right] \times\right. \\
& \left.\times\left[\left(\hat{X}_{B}^{\phi^{1}}\right)^{\frac{1}{3}}+i\left(\hat{X}_{B}^{\phi^{2}}\right)^{\frac{1}{3}}+j\left(\hat{X}_{B}^{\phi^{3}}\right)^{\frac{1}{3}}+k\left(\hat{X}_{B}^{\phi^{4}}\right)^{\frac{1}{3}}\right]\right\rangle\left.\right|^{2} \\
= & \mid\left\langle\left[\left(\hat{X}_{A}^{\theta^{1}}\right)^{\frac{1}{3}}\left(\hat{X}_{B}^{\phi^{1}}\right)^{\frac{1}{3}}-\left(\hat{X}_{A}^{\theta^{2}}\right)^{\frac{1}{3}}\left(\hat{X}_{B}^{\phi^{2}}\right)^{\frac{1}{3}}\right.\right. \\
& \left.-\left(\hat{X}_{A}^{\theta^{3}}\right)^{\frac{1}{3}}\left(\hat{X}_{B}^{\phi^{3}}\right)^{\frac{1}{3}}-\left(\hat{X}_{A}^{\theta^{4}}\right)^{\frac{1}{3}}\left(\hat{X}_{B}^{\phi^{4}}\right)^{\frac{1}{3}}\right] \\
& +i\left[\left(\hat{X}_{A}^{\theta^{2}}\right)^{\frac{1}{3}}\left(\hat{X}_{B}^{\phi^{1}}\right)^{\frac{1}{3}}+\left(\hat{X}_{A}^{\theta^{1}}\right)^{\frac{1}{3}}\left(\hat{X}_{B}^{\phi^{2}}\right)^{\frac{1}{3}}\right. \\
& \left.-\left(\hat{X}_{A}^{\theta^{4}}\right)^{\frac{1}{3}}\left(\hat{X}_{B}^{\phi^{3}}\right)^{\frac{1}{3}}+\left(\hat{X}_{A}^{\theta^{3}}\right)^{\frac{1}{3}}\left(\hat{X}_{B}^{\phi^{4}}\right)^{\frac{1}{3}}\right] \\
& +j\left[\left(\hat{X}_{A}^{\theta^{3}}\right)^{\frac{1}{3}}\left(\hat{X}_{B}^{\phi^{1}}\right)^{\frac{1}{3}}+\left(\hat{X}_{A}^{\theta^{4}}\right)^{\frac{1}{3}}\left(\hat{X}_{B}^{\phi^{2}}\right)^{\frac{1}{3}}\right. \\
& \left.+\left(\hat{X}_{A}^{\theta^{1}}\right)^{\frac{1}{3}}\left(\hat{X}_{B}^{\phi^{3}}\right)^{\frac{1}{3}}-\left(\hat{X}_{A}^{\theta^{2}}\right)^{\frac{1}{3}}\left(\hat{X}_{B}^{\phi^{4}}\right)^{\frac{1}{3}}\right] \\
& +k\left[\left(\hat{X}_{A}^{\theta^{4}}\right)^{\frac{1}{3}}\left(\hat{X}_{B}^{\phi^{1}}\right)^{\frac{1}{3}}-\left(\hat{X}_{A}^{\theta^{3}}\right)^{\frac{1}{3}}\left(\hat{X}_{B}^{\phi^{2}}\right)^{\frac{1}{3}}\right. \\
& \left.\left.+\left(\hat{X}_{A}^{\theta^{2}}\right)^{\frac{1}{3}}\left(\hat{X}_{B}^{\phi^{3}}\right)^{\frac{1}{3}}+\left(\hat{X}_{A}^{\theta^{1}}\right)^{\frac{1}{3}}\left(\hat{X}_{B}^{\phi^{4}}\right)^{\frac{1}{3}}\right]\right\rangle\left.\right|^{2},
\end{aligned}
$$

Similarly, the RHS is:

$$
\begin{aligned}
R H S= & \left\langle\left\{\left(\hat{X}_{A}^{\theta^{1}}\right)^{2 / 3}+\left(\hat{X}_{A}^{\theta^{2}}\right)^{2 / 3}+\left(\hat{X}_{A}^{\theta^{3}}\right)^{2 / 3}+\left(\hat{X}_{A}^{\theta^{4}}\right)^{2 / 3}\right\}\right. \\
& \left.\left\{\left(\hat{X}_{B}^{\phi^{1}}\right)^{2 / 3}+\left(\hat{X}_{B}^{\phi^{2}}\right)^{2 / 3}+\left(\hat{X}_{B}^{\phi^{3}}\right)^{2 / 3}+\left(\hat{X}_{B}^{\phi^{4}}\right)^{2 / 3}\right\}\right\rangle \\
= & \left\langle\left(\hat{X}_{A}^{\theta^{1}}\right)^{2 / 3}\left(\hat{X}_{B}^{\phi^{1}}\right)^{2 / 3}+\left(\hat{X}_{A}^{\theta^{1}}\right)^{2 / 3}\left(\hat{X}_{B}^{\phi^{2}}\right)^{2 / 3}\right. \\
& +\left(\hat{X}_{A}^{\theta^{1}}\right)^{2 / 3}\left(\hat{X}_{B}^{\phi^{3}}\right)^{2 / 3}+\left(\hat{X}_{A}^{\theta^{1}}\right)^{2 / 3}\left(\hat{X}_{B}^{\phi^{4}}\right)^{2 / 3} \\
& +\left(\hat{X}_{A}^{\theta^{2}}\right)^{2 / 3}\left(\hat{X}_{B}^{\phi^{1}}\right)^{2 / 3}+\left(\hat{X}_{A}^{\theta^{2}}\right)^{2 / 3}\left(\hat{X}_{B}^{\phi^{2}}\right)^{2 / 3} \\
& +\left(\hat{X}_{A}^{\theta^{2}}\right)^{2 / 3}\left(\hat{X}_{B}^{\phi^{3}}\right)^{2 / 3}+\left(\hat{X}_{A}^{\theta^{2}}\right)^{2 / 3}\left(\hat{X}_{B}^{\phi^{4}}\right)^{2 / 3} \\
& +\left(\hat{X}_{A}^{\theta^{3}}\right)^{2 / 3}\left(\hat{X}_{B}^{\phi^{1}}\right)^{2 / 3}+\left(\hat{X}_{A}^{\theta^{3}}\right)^{2 / 3}\left(\hat{X}_{B}^{\phi^{2}}\right)^{2 / 3} \\
& +\left(\hat{X}_{A}^{\theta^{3}}\right)^{2 / 3}\left(\hat{X}_{B}^{\phi^{3}}\right)^{2 / 3}+\left(\hat{X}_{A}^{\theta^{3}}\right)^{2 / 3}\left(\hat{X}_{B}^{\phi^{4}}\right)^{2 / 3} \\
& +\left(\hat{X}_{A}^{\theta^{4}}\right)^{2 / 3}\left(\hat{X}_{B}^{\phi^{1}}\right)^{2 / 3}+\left(\hat{X}_{A}^{\theta^{4}}\right)^{2 / 3}\left(\hat{X}_{B}^{\phi^{2}}\right)^{2 / 3} \\
& \left.+\left(\hat{X}_{A}^{\theta^{4}}\right)^{2 / 3}\left(\hat{X}_{B}^{\phi^{3}}\right)^{2 / 3}+\left(\hat{X}_{A}^{\theta^{4}}\right)^{2 / 3}\left(\hat{X}_{B}^{\phi^{4}}\right)^{2 / 3}\right\rangle,(6.3)
\end{aligned}
$$

where we denote sites $n=1,2$ as $A, B$ respectively, with phase angles $\theta_{1}^{l}=\theta^{l}$ and $\theta_{2}^{l}=\phi^{l}$. The averages of $\left\langle\left(\hat{X}_{A}^{\theta^{l}}\right)^{\frac{1}{3}}\left(\hat{X}_{B}^{\phi^{l}}\right)^{\frac{1}{3}}\right\rangle=0.433881 \cos \left(\phi^{l}-\theta^{l}\right)$ and
$\left\langle\left(\hat{X}_{A}^{\theta^{l}}\right)^{\frac{2}{3}}\left(\hat{X}_{B}^{\phi^{l}}\right)^{\frac{2}{3}}\right\rangle=0.42583$ are obtained following the methods given in the Appendix. We find the value of the $L H S$ of Shchukin and Vogel's inequality is

$$
\begin{aligned}
L H S= & \mid 0.434881\left\{\left[\cos \left(\phi^{1}-\theta^{1}\right)-\cos \left(\phi^{2}-\theta^{2}\right)\right.\right. \\
& \left.-\cos \left(\phi^{3}-\theta^{3}\right)-\cos \left(\phi^{4}-\theta^{4}\right)\right] \\
& +i\left[\cos \left(\phi^{1}-\theta^{2}\right)+\cos \left(\phi^{2}-\theta^{1}\right)\right. \\
& \left.-\cos \left(\phi^{3}-\theta^{4}\right)+\cos \left(\phi^{4}-\theta^{3}\right)\right] \\
& +j\left[\cos \left(\phi^{1}-\theta^{3}\right)+\cos \left(\phi^{2}-\theta^{4}\right)\right. \\
& \left.+\cos \left(\phi^{3}-\theta^{1}\right)-\cos \left(\phi^{4}-\theta^{2}\right)\right] \\
& +k\left[\cos \left(\phi^{1}-\theta^{4}\right)-\cos \left(\phi^{4}-\theta^{3}\right)\right. \\
& \left.\left.+\cos \left(\phi^{3}-\theta^{2}\right)+\cos \left(\phi^{4}-\theta^{1}\right)\right]\right\}\left.\right|^{2},
\end{aligned}
$$

using angles like $\theta^{1}=\phi^{1}=0$, and $\theta^{2}=\theta^{3}=\theta^{4}=-\phi^{2}=$ $-\phi^{3}=-\phi^{4}=-\pi / 2$, to get $L H S=|0.434881 \times 4|^{2}$ as all imaginary values are 0 . There are many optimal choice of angles, but the maximum values of the $L H S$ are same. The value of the $R H S$ is

$$
\begin{aligned}
R H S & =16\left\langle\left(\hat{X}_{A}^{\theta^{1}}\right)^{2 / 3}\left(\hat{X}_{B}^{\phi^{1}}\right)^{2 / 3}\right\rangle \\
& =0.42583 \times 16,
\end{aligned}
$$

so that the Bell ratio is the same as with the functional CFRD approach, using a power law with $m=1 / 3$ :

$$
\begin{aligned}
B_{2-S V(4)} & =\frac{|0.434881 \times 4|^{2}}{0.42583 \times 16} \\
& =\frac{|0.434881 \times 2|^{2}}{0.42583 \times 4} \\
& =B_{2-C F R D .}
\end{aligned}
$$

This is caused by the optimal value of the phases corresponding effectively to only two observables per site, since three of those phases have the same value.

\section{Four site case}

Next, we check mode number $N=4$, and $r=2$, for states like $|\psi\rangle=1 / \sqrt{2}(|0011\rangle+|1100\rangle)$. In QM, the operators at each site in Shchukin and Vogel's theory are

$$
\begin{aligned}
& \hat{F}_{A}=\left(\hat{X}_{A}^{\theta^{1}}\right)^{m}+i\left(\hat{X}_{A}^{\theta^{2}}\right)^{m}+j\left(\hat{X}_{A}^{\theta^{3}}\right)^{m}+k\left(\hat{X}_{A}^{\theta^{4}}\right)^{m}, \\
& \hat{F}_{B}=\left(\hat{X}_{B}^{\phi^{1}}\right)^{m}+i\left(\hat{X}_{B}^{\phi^{2}}\right)^{m}+j\left(\hat{X}_{B}^{\phi^{3}}\right)^{m}+k\left(\hat{X}_{B}^{\phi^{4}}\right)^{m}, \\
& \hat{F}_{C}=\left(\hat{X}_{C}^{\gamma^{1}}\right)^{m}+i\left(\hat{X}_{C}^{\gamma^{2}}\right)^{m}+j\left(\hat{X}_{C}^{\gamma^{3}}\right)^{m}+k\left(\hat{X}_{C}^{\gamma^{4}}\right)^{m}, \\
& \hat{F}_{D}=\left(\hat{X}_{D}^{\varphi^{1}}\right)^{m}+i\left(\hat{X}_{D}^{\varphi^{2}}\right)^{m}+j\left(\hat{X}_{D}^{\varphi^{3}}\right)^{m}+k\left(\hat{X}_{D}^{\varphi^{4}}\right)^{m},
\end{aligned}
$$

where again $\theta_{1}^{l}=\theta^{l}, \theta_{2}^{l}=\phi^{l}, \theta_{3}^{l}=\gamma^{l}$, and $\theta_{4}^{l}=\varphi^{l}$. After extensive algebra, we find that the largest Bell values with optimal choice of angles are still the same as in the CFRD case using $m=1 / 3: B_{4-S V(4)}=B_{4-C F R D}$. The reason for this identity, as in the two-site case, is that with optimal settings we have effectively only two measurements per site. 


\section{B. Eight setting example}

Finally, we can check the SV inequality with 8 observables at each site. This approach uses octonions, and results in a rather complex inequality [19]. Therefore, we only consider here the simplest two-site case $|\psi\rangle=c_{1}|0\rangle|1\rangle+c_{2}|1\rangle|0\rangle, c_{1}=c_{2}=1 / \sqrt{2}$.

We consider the fractional case $m=1 / 3$ as an example. The LHS of the inequality is:

$$
\begin{aligned}
\left|\left\langle\hat{F}_{A} \hat{F}_{B}\right\rangle\right|^{2}= & \mid 0.434881\left\{\left[\cos \left(\phi^{1}-\theta^{1}\right)-\cos \left(\phi^{2}-\theta^{2}\right)\right.\right. \\
& -\cos \left(\phi^{3}-\theta^{3}\right)-\cos \left(\phi^{4}-\theta^{4}\right) \\
& -\cos \left(\phi^{5}-\theta^{5}\right)-\cos \left(\phi^{6}-\theta^{6}\right) \\
& \left.-\cos \left(\phi^{7}-\theta^{7}\right)-\cos \left(\phi^{8}-\theta^{8}\right)\right] \\
& +i_{1}\left[\cos \left(\phi^{1}-\theta^{2}\right)+\cos \left(\phi^{2}-\theta^{1}\right)\right. \\
& +\cos \left(\phi^{3}-\theta^{4}\right)-\cos \left(\phi^{4}-\theta^{3}\right) \\
& +\cos \left(\phi^{5}-\theta^{6}\right)-\cos \left(\phi^{6}-\theta^{5}\right) \\
& \left.-\cos \left(\phi^{7}-\theta^{8}\right)+\cos \left(\phi^{8}-\theta^{7}\right)\right] \\
& +i_{2}[\cdots]+i_{3}[\cdots]+i_{4}[\cdots]+i_{5}[\cdots] \\
& \left.+i_{6}[\cdots]+i_{7}[\cdots]\right\}\left.\right|^{2},
\end{aligned}
$$

where $\hat{F}_{A}=\left(\hat{X}_{A}^{\theta^{1}}\right)^{m}+i_{1}\left(\hat{X}_{A}^{\theta^{2}}\right)^{m}+i_{2}\left(\hat{X}_{A}^{\theta^{3}}\right)^{m}+i_{3}\left(\hat{X}_{A}^{\theta^{4}}\right)^{m}+$ $i_{4}\left(\hat{X}_{A}^{\theta^{5}}\right)^{m}+i_{5}\left(\hat{X}_{A}^{\theta^{6}}\right)^{m}+i_{6}\left(\hat{X}_{A}^{\theta^{7}}\right)^{m}+i_{7}\left(\hat{X}_{A}^{\theta^{8}}\right)^{m}$, is an octonionic function of real observables, $\left(\hat{X}_{A}^{\theta^{l}}\right)^{m},(l=1-8)$ at site $\mathrm{A}$; similarly for site $\mathrm{B}$, involving $\left(\hat{X}_{B}^{\phi^{l}}\right)^{m}$. The multiplication rules for the imaginary units of octonions were listed in [19]. One of the optimal choices of angles still keeps the same pattern as in two-sites case: $\theta^{1}=\phi^{1}=0$, and $\theta^{l \neq 1}=-\phi^{l \neq 1}=-\pi / 2$, so that we obtain $L H S=|0.434881 \times 8|^{2}$, and

$$
R H S=64\left\langle\left(\hat{X}_{A}^{\theta^{1}}\right)^{2 / 3}\left(\hat{X}_{B}^{\phi^{1}}\right)^{2 / 3}\right\rangle .
$$

Therefore

$$
B_{2-S V(8)}=\frac{|0.434881 \times 8|^{2}}{0.4258 \times 64}=B_{2-C F R D .}
$$

For $N=4$ modes, the optimal result of the SV inequality with 8 observables at each site is also the same as for the functional CFRD fractional moment inequality, for the particular state $|\psi\rangle=1 / \sqrt{2}(|0011\rangle+|1100\rangle)$.

In summary, in the cases investigated, the results are the same as those found with the simpler CFRD approach, using fractional moments. The reason is that the optimal values can be found with only 2 effective settings per site with the types of states we consider. However, this situation may change with more general input states or functional transformations. We leave this as an open question for future work.

\section{SENSITIVITY TO LOSS AND MIXING}

The value of the Bell observable $B_{N}$ or $S_{N}$ increases with the number of sites $N$, and this is suggestive of a strategy that may allow genuine loophole-free violations of LR. However, it may be argued that since increasing the number of sites will increase the number of detectors required, there will be no advantage. Only careful calculation of the Bell observable $B_{N}$ including the detection efficiency $\eta$ can determine whether the strategy is advantageous for loophole-free violation of Bell inequalities.

\section{A. Model of loss and impurity}

Loss is modelled using a beam splitter as follows. The field modes $\hat{a}_{i n}$ at each site are independently coupled to a second mode $\hat{a}_{v}$, assumed to be in a vacuum state. Bosons are lost from the field into the vacuum mode, the strength of coupling determining the rate of loss. For each mode, the beam splitter model gives the final detected and vacuum mode in terms of the inputs $\hat{a}_{i n}$ and $\hat{a}_{v}$

$$
\begin{aligned}
\hat{a}_{\text {out }} & =\sqrt{\eta} \hat{a}_{\text {in }}+\sqrt{1-\eta} \hat{a}_{v}, \\
\hat{a}_{v, \text { out }} & =\sqrt{1-\eta} \hat{a}_{\text {in }}-\sqrt{\eta} \hat{a}_{v},
\end{aligned}
$$

where $\eta$ is the efficiency, the probability for detecting a boson, given that one is incident. One can rewrite these relations to obtain

$$
\begin{aligned}
\hat{a}_{\text {in }} & =\sqrt{\eta} \hat{a}_{\text {out }}+\sqrt{1-\eta} \hat{a}_{v, \text { out }}, \\
\hat{a}_{v} & =\sqrt{1-\eta} \hat{a}_{\text {out }}-\sqrt{\eta} \hat{a}_{v, \text { out }} .
\end{aligned}
$$

Since we only measure " $\hat{a}_{\text {out }}$ ", not " $\hat{a}_{v, \text { out }}$ ", we need to trace over all the unmeasured vacuum output modes to obtain the final density operator for the detected modes after loss.

Thus, given a total density matrix of $\hat{\rho}$, we only measure a reduced density matrix $\hat{\rho}_{\text {out }}$, where:

$$
\begin{aligned}
\hat{\rho}_{\text {out }} & =\operatorname{Tr}_{v, \text { out }}\{\hat{\rho}\} \\
& =\sum_{k}\left\langle\left.\psi_{k}\right|_{v, \text { out }} \hat{\rho} \mid \psi_{k}\right\rangle_{v, \text { out }}
\end{aligned}
$$

We also examine the effect of impurity, by considering an input state $\hat{\rho}^{\prime}=p|\psi\rangle\langle\psi|+(1-p) \hat{\rho}_{\text {mix }}$, where $\hat{\rho}_{\text {mix }}$ is the mixed state obtained by complete decoherence in the occupation-number basis, i.e.

$$
\begin{aligned}
\hat{\rho}_{\text {mix }}= & \left|c_{1}\right|^{2}|0\rangle^{\otimes r}|1\rangle^{\otimes(N-r)}\left\langle0 | ^ { \otimes r } \left\langle\left. 1\right|^{\otimes(N-r)}+\right.\right. \\
& +\left|c_{2}\right|^{2}|1\rangle^{\otimes r}|0\rangle^{\otimes(N-r)}\left\langle1 | ^ { \otimes r } \left\langle\left. 0\right|^{\otimes(N-r)} .\right.\right.
\end{aligned}
$$

Here $p$ is the probability the system is in the original pure state (3.4). While this is not the most general model of state impurity, it is a relatively simple one that allows us to carry out indicative calculations of the effects of impure input states.

\section{B. Three-mode MABK example}

We can transform the input state for $N=3$ and $r=1$, $|\psi\rangle=\frac{1}{\sqrt{2}}(|100\rangle+|011\rangle)|000\rangle$, for example, where $|000\rangle$ 
represents the vacuum fields, into the output after loss. We denote the three field mode operators involved as $\hat{a}$, $\hat{b}, \hat{c}$ for clarity. Thus in this case,

$$
\begin{aligned}
\mid \text { out }\rangle= & {\left[\hat{a}_{\text {in }}^{\dagger}|000\rangle|000\rangle+\hat{b}_{\text {in }}^{\dagger} \hat{c}_{\text {in }}^{\dagger}|000\rangle|000\rangle\right] / \sqrt{2} } \\
= & \left(\sqrt{\eta}|100\rangle_{\text {out }}|000\rangle_{v, \text { out }}+\right. \\
& \sqrt{1-\eta}|000\rangle_{\text {out }}|100\rangle_{v, \text { out }}+\eta|011\rangle_{\text {out }}|000\rangle_{v, \text { out }} \\
& +(1-\eta)|000\rangle_{\text {out }}|011\rangle_{v, \text { out }}+ \\
& \sqrt{1-\eta} \sqrt{\eta}\left(|001\rangle_{\text {out }}|010\rangle_{v, \text { out }}\right. \\
& \left.+|010\rangle_{\text {out }}|001\rangle_{v, \text { out }}\right) / \sqrt{2} .
\end{aligned}
$$

Next, we need to trace over the vacuum output modes to obtain a reduced density matrix $\hat{\rho}_{\text {out }}$, where:

$$
\begin{aligned}
\hat{\rho}_{\text {out }}= & \operatorname{Tr}_{v, \text { out }}\{\hat{\rho}\} \\
= & \sum_{i, j, k}\left\langle\left. i j k\right|_{v, \text { out }} \hat{\rho} \mid i j k\right\rangle_{v, \text { out }} \\
= & \frac{1}{2}\{[\sqrt{\eta}|100\rangle+\eta|011\rangle][\sqrt{\eta}\langle 100|+\eta\langle 011|]+ \\
& (1-\eta)|000\rangle\langle 000|+\eta(1-\eta)| 001\rangle\langle 001| \\
& +\eta(1-\eta)|010\rangle\langle 010|+ \\
& \left.+(1-\eta)^{2}|000\rangle\langle 000|\right\} .
\end{aligned}
$$

Proceeding, the probability distributions are evaluated. Thus, in this three-mode example:

$$
\begin{aligned}
P\left(x_{\theta}, x_{\phi}, x_{\gamma}\right)= & \left\langle x_{\theta}\left|\left\langle x_{\phi}\left|\left\langle x_{\gamma}\left|\hat{\rho}_{\text {out }}\right| x_{\theta}\right\rangle\right| x_{\phi}\right\rangle\right| x_{\gamma}\right\rangle \\
= & \frac{1}{2}\left(\frac{2}{\pi}\right)^{3 / 2} e^{-2\left(x_{\theta}^{2}+x_{\phi}^{2}+x_{\gamma}^{2}\right)} \times \\
& \times\left[\left|\left(2 \sqrt{\eta} e^{i \theta} x_{\theta}+4 \eta e^{i \phi} e^{i \gamma} x_{\phi} x_{\gamma}\right)\right|^{2}+\right. \\
& +4 \eta(1-\eta) \times\left\{\left\{\left|x_{\gamma}\right|^{2}+\left|x_{\phi}\right|^{2}\right\}\right. \\
& \left.+\left[1-\eta+(1-\eta)^{2}\right]\right],
\end{aligned}
$$

and the integrals give for example

$$
\left\langle\hat{X}_{A}^{\theta, b i n} \hat{X}_{B}^{\phi, b i n} \hat{X}_{C}^{\gamma, b i n}\right\rangle=\left(\frac{2 \eta}{\pi}\right)^{\frac{3}{2}} \cos (\gamma+\phi-\theta)(7.8
$$

Examining the MABK Bell inequalities defined by (4.2), we finally obtain, using the "orthogonal" choice of measurements given for (4.7), the result:

$$
\left|\left\langle\hat{a}_{b i n}^{\dagger} \hat{b}_{b i n} \hat{c}_{b i n}\right\rangle\right|=4\left(\frac{2 \eta}{\pi}\right)^{\frac{3}{2}} .
$$

Alternatively, for the measurement angles $\theta=0, \phi=$ $-\pi / 6, \gamma=-2 \pi / 6, \theta^{\prime}=\pi / 2, \phi^{\prime}=-4 \pi / 6, \gamma^{\prime}=-5 \pi / 6$, we can rotate the $L H S$ moment, but so that again

$$
\left|\left\langle\hat{a}_{b i n}^{\dagger} \hat{b}_{b i n} \hat{c}_{b i n}\right\rangle\right|=4\left(\frac{2 \eta}{\pi}\right)^{\frac{3}{2}}
$$

\section{Higher $\mathrm{N}$ results for MABK inequalities}

Continuing in this fashion for higher $N$, we find that the values of the Bell observable including the effect of detection inefficiencies with the optimal choice of angles is

$$
S_{N}=\frac{1}{\sqrt{2}}\left(\frac{4 \eta}{\pi}\right)^{\frac{N}{2}},
$$

which implies a critical minimum efficiency

$$
\eta_{\min }=2^{(1-2 N) / N} \pi
$$

in order to violate the MABK inequalities (4.2).

We can also examine the effect of impurity or noise, by considering a state $\hat{\rho}^{\prime}=p|\psi\rangle\langle\psi|+(1-p) \hat{\rho}_{\text {mix }}$, as described above. The effect of this type of 'noise' on the MABK inequality is to give

$$
S_{N}(p, \eta)=\frac{\sqrt{2}}{2}\left(\frac{4 \eta p^{2}}{\pi}\right)^{\frac{N}{2}} .
$$

This result agrees with that obtained by Acin et al [15] for the case $\eta=1$.

For lower $N$, the strategy of binning and using the MABK inequalities shows an advantage, by allowing a violation of LHV theories for $N=3,4,5$. However, very high efficiencies, $\eta>0.99,0.93,0.90$ respectively, even for a pure state $p=1$, are required, as shown in Fig. 5. While high detection efficiencies are feasible for homodyne detection, these efficiency values are still quite challenging once generation losses are also taken into account. In view of this, the high requirement for $\eta_{\min }$ for the case $N=3$ may be prohibitive.

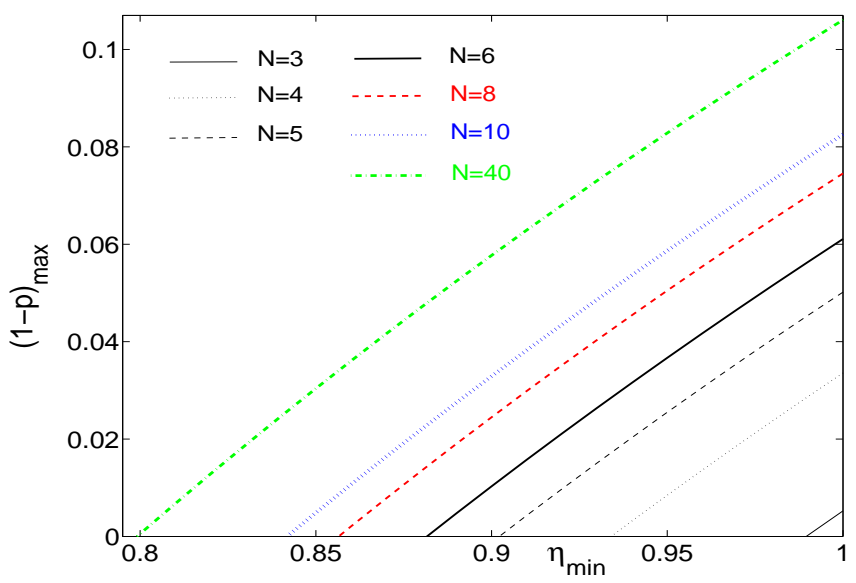

Figure 5: (Color online) The boundary of maximum noise $(1-p)_{\max }$ and minimum detection efficiency $\eta_{\min }$ to violate the MABK Bell inequality with binned outcomes. Different $N$ is distinguished by the line style. 


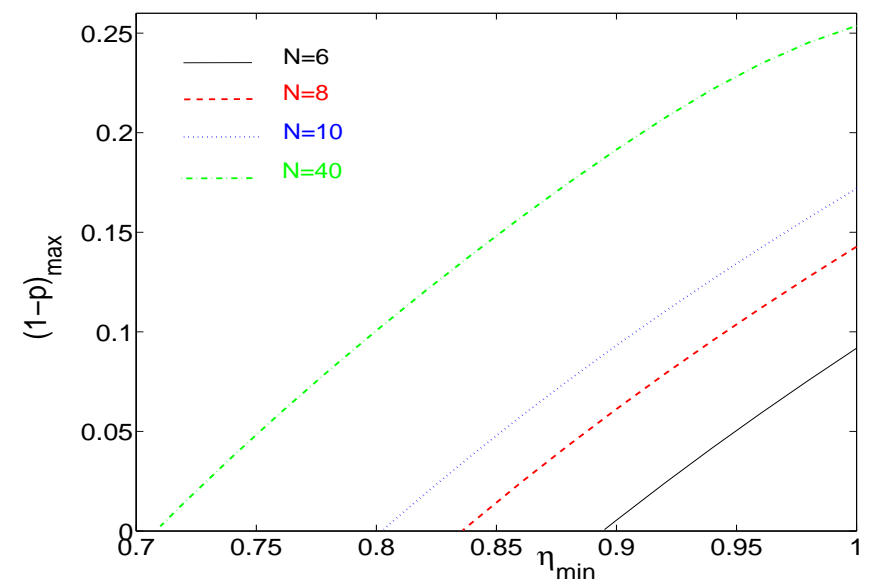

Figure 6: (Color online) The boundary of maximum noise $(1-p)_{\max }$ and minimum detection efficiency $\eta_{\min }$ to violate the functional CFRD Bell inequality with observables as the optimal function. Different $N$ is distinguished by the line style.

\section{Loss and noise with functional inequalities}

This approach is also applied to enable a prediction of the effect of loss and noise on the functional inequalities of Section IV, and the results are plotted in Fig. 6] for the optimum function. Here, the parameter $\varepsilon_{N}$ is changed to $\varepsilon_{N}(\eta)$ for the optimum function. For $N$ even we find that

$$
\begin{aligned}
\varepsilon_{N}(\eta) & =\frac{2 \eta \varepsilon_{N}}{2 \eta+(1-\eta) \varepsilon_{N}} \\
B_{N} & =2^{N-2}\left[\frac{2\left(I^{+}\right)^{4}(\eta p)^{2}}{\pi I^{o} C}\right]^{\frac{N}{2}}
\end{aligned}
$$

where $\varepsilon_{N}$ is defined as before, and $C=\eta I+(1-\eta) I^{o}$. For the case of odd $N$ the relevant integral equations change, giving a modified (and slightly reduced) Bell variable $B_{N}^{\prime}$, where:

$$
\begin{aligned}
\varepsilon_{N}^{\prime}(\eta) & =\varepsilon_{N}(\eta) \frac{N \varepsilon_{N}^{+}(\eta)-\varepsilon_{N}(\eta) \varepsilon_{N}^{-} / \varepsilon_{N}}{N \varepsilon_{N}^{+}(\eta)+\varepsilon_{N}^{2}(\eta) \varepsilon_{N}^{-} / \varepsilon_{N}^{2}} \\
B_{N}^{\prime} & =\frac{2 \sqrt{I^{o} C}}{I^{o}+C} B_{N} .
\end{aligned}
$$

Here $\varepsilon_{N}^{+}(\eta)=\varepsilon_{N}(\eta)+4$, and $B_{N}$ is defined as in Eq (7.14).

For pure state, in the case of $r=N / 2, N$ is even, we can give the threshold efficiency requirement analytically since all integrals are independent of $N$, where

$$
\eta_{\text {crit }}=\frac{\left[\left(4-\varepsilon_{N}\right)+\sqrt{\left(4-\varepsilon_{N}\right)^{2}+4 \varepsilon_{N}^{2}}\right] I_{0} I \pi}{2^{6-4 / N}\left(I^{+}\right)^{4}} .
$$

This reduces at large $N$ to an asymptotic value of $\eta_{\infty}=$ 0.69 , thus dramatically reducing the required detection efficiency. The violation is less sensitive to detector inefficiency in the macroscopic, large $N$ limit.

Compared with the MABK binning approach, the robustness of the functional inequality against noise and inefficiency is apparent as shown in Fig. 6. For $N>7$, the functional CV inequalities used with an optimal function shows clear advantages, allowing violation of LHV at much lower efficiencies and larger maximum noise. For example, for $N=40$, we can get Bell violation with $\eta_{\text {crit }} \sim 70.8 \%$ for pure state, or $p_{\max } \sim 0.25$ for ideal detection efficiency. The potentially feasible $\eta_{\text {crit }} \sim 80 \%$ requires $N \approx 10$ for a pure state if the functional optimization is used. By comparison, the binned case requires $N \sim 40$, and the original $\mathrm{CFRD}$ cannot reach this even with $N \rightarrow \infty$.

\section{CONCLUSION}

In summary, we have developed a functional approach to tests of quantum nonlocality, and showed how this gives an exceptional degree of robustness against losses and noise. For continuous variable measurements on GHZ states, this approach gives distinct advantages when compared with either binning methods or the momentbased approach of Cavalcanti et al. (CFRD) [18]. We have also compared the class of inequalities derived by CFRD with the generalizations given by Shchukin and Vogel (SV) for more observables at each site but found no better violations with the photon-number correlated states introduced here.

However, there are still many open questions. The first is applicable even to the CFRD result: we do not know yet if there are any other quantum states that can give better violations than those given here for the GHZ-type states. Another question is whether there are other states for which the multiple-observable SV inequalities provide an advantage over the CFRD. One may also ask if there are yet more general continuous variable inequalities than either the CFRD or SV results.

Further applications of these ideas may include application of the functional approach to tests of other forms of nonlocality - e.g, entanglement and EPR-steering 33. It seems possible that a functional optimization will allow more robust implementations of these tests as well.

\section{ACKNOWLEDGEMENT}

We wish to acknowledge funding for this project from the Australian Research Council through a Discovery grant and the Australian Research Council Center of Excellence for Quantum-Atom Optics. 


\section{Appendix I: Quadrature Wavefunctions}

Calculations with the functional transformations used here rely on the fact that quadrature variables have the same wave-functions as the quantum harmonic oscillator. We first recall that the harmonic oscillator Hamiltonian with unit mass and frequency $\omega$ can be written:

$$
\hat{H}=\frac{1}{2}\left(\hat{p}^{2}+\omega^{2} \hat{x}^{2}\right) .
$$

Here $\hat{p}$ is the momentum and $\hat{x}$ is the position operator, with commutators $[\hat{x}, \hat{p}]=i \hbar$, and creation and annihilation operators defined as:

$$
\begin{aligned}
\hat{a} & =(2 \hbar \omega)^{-1 / 2}(\omega \hat{q}+i \hat{p}), \\
\hat{a}^{\dagger} & =(2 \hbar \omega)^{-1 / 2}(\omega \hat{q}-i \hat{p}) .
\end{aligned}
$$

For simplicity we assume that $\omega=1$ and $\hbar=1 / 2$, which corresponds to the convention used throughout the paper:

$$
\begin{aligned}
& \hat{x}=\left(\hat{a}^{\dagger}+\hat{a}\right) / 2, \\
& \hat{p}=-i\left(\hat{a}-\hat{a}^{\dagger}\right) / 2 .
\end{aligned}
$$

\section{A. Wavefunction for quadrature variables}

In this standard form, the harmonic oscillator wavefunction 34] for the $n^{\text {th }}$ energy eigenstate is:

$$
\langle x \mid n\rangle=\left(\frac{2}{\pi 2^{2 n-1}[n !]^{2}}\right)^{1 / 4} H_{n}(\sqrt{2} x) e^{-x^{2}},
$$

where $H_{n}(x)$ is the $n^{\text {th }}$ Hermite polynomial. Thus, for example:

$$
\begin{aligned}
& \langle x \mid 0\rangle=\left(\frac{2}{\pi}\right)^{1 / 4} e^{-x^{2}}, \\
& \langle x \mid 1\rangle=2 x\left(\frac{2}{\pi}\right)^{1 / 4} e^{-x^{2}} .
\end{aligned}
$$

We also need the complementary (momentum) wavefunctions:

$$
\langle p \mid \psi\rangle=\frac{1}{\sqrt{\pi}} \int_{-\infty}^{\infty} \exp \left(-2 i p^{\prime} x^{\prime}\right)\left\langle x^{\prime} \mid \psi\right\rangle d x^{\prime},
$$

Again, using normalized quadrature variables, one finds that:

$$
\begin{aligned}
& \langle p \mid 0\rangle=\left(\frac{2}{\pi}\right)^{1 / 4} e^{-p^{2}}, \\
& \langle p \mid 1\rangle=-2 i p\left(\frac{2}{\pi}\right)^{1 / 4} e^{-p^{2}} .
\end{aligned}
$$

\section{B. General rotation}

Next, we consider the case of a general rotation of the quadrature phase by defining

$$
\hat{X}^{\theta}=\hat{x} \cos \theta+\hat{p} \sin \theta=\left(\hat{a} e^{-i \theta}+\hat{a}^{\dagger} e^{i \theta}\right) / 2,
$$

Hence we find that

$$
\begin{aligned}
& \left\langle X^{\theta} \mid 0\right\rangle=\left(\frac{2}{\pi}\right)^{1 / 4} e^{-\left(X^{\theta}\right)^{2}}, \\
& \left\langle X^{\theta} \mid 1\right\rangle=2 e^{-i \theta}\left(\frac{2}{\pi}\right)^{1 / 4} X^{\theta} e^{-\left(X^{\theta}\right)^{2}},
\end{aligned}
$$

and $p$ corresponds to $\theta=\pi / 2$.

\section{Joint probabilities}

To carry out moment calculations, it is necessary to have joint probabilities. As an example of this calculation, we consider a two-mode state $\hat{\rho}=|\Psi\rangle\langle\Psi|$, where:

$$
|\Psi\rangle=\frac{1}{\sqrt{2}}[|0,1\rangle+|1,0\rangle] .
$$

Carrying out the calculation of the joint probability, and defining $|\mathbf{X}|^{2}=\left(X^{\theta}\right)^{2}+\left(X^{\phi}\right)^{2}$, we find that:

$$
\begin{aligned}
P\left(X^{\theta}, X^{\phi}\right)= & \left\langle X^{\theta}\left|\left\langle X^{\phi}|\hat{\rho}| X^{\theta}\right\rangle\right| X^{\phi}\right\rangle \\
= & \frac{1}{2}\left|\left\langle X^{\theta} \mid 0\right\rangle\left\langle X^{\phi} \mid 1\right\rangle+\left\langle X^{\theta} \mid 1\right\rangle\left\langle X^{\phi} \mid 0\right\rangle\right|^{2} \\
= & \left(\frac{4}{\pi}\right)\left|e^{-|\mathbf{X}|^{2}}\left(e^{i \phi} X^{\phi}+e^{i \theta} X^{\theta}\right)\right|^{2} \\
= & \left(\frac{4}{\pi}\right) e^{-2|\mathbf{X}|^{2}} \times \\
& \times\left[|\mathbf{X}|^{2}+2 X^{\theta} X^{\phi} \cos (\theta-\phi)\right]
\end{aligned}
$$

\section{Binned moment calculation}

As an example of the application of the joint probability, consider a binned moment calculation for the case $N=2$, using an initial Bell state of the form given above. Then we obtain:

$$
\begin{aligned}
\left\langle\hat{X}_{A}^{\theta, \text { bin }} \hat{X}_{B}^{\phi, b i n}\right\rangle= & \int_{X^{\theta}=0}^{\infty} \int_{X^{\phi}=0}^{\infty} P\left(X^{\theta}, X^{\phi}\right) d^{2} \mathbf{X} \\
& -\int_{X^{\theta}=0}^{\infty} \int_{X^{\phi}=-\infty}^{0} P\left(X^{\theta}, X^{\phi}\right) d^{2} \mathbf{X} \\
& +\int_{X^{\theta}=-\infty}^{0} \int_{X^{\phi}=-\infty}^{0} P\left(X^{\theta}, X^{\phi}\right) d^{2} \mathbf{X} \\
& -\int_{X^{\theta}=-\infty}^{0} \int_{X^{\phi}=0}^{\infty} P\left(X^{\theta}, X^{\phi}\right) d^{2} \mathbf{X} \\
= & \left(\frac{2}{\pi}\right) \cos (\phi-\theta) .
\end{aligned}
$$




\section{E. Functional moment calculation}

Here we show how to calculate the expectation value of products of functional moments using the joint probabilities. For the case $N=2$, using the same state given above, the value of the LHS is

$$
L H S=\left|\left\langle\prod_{n=1}^{2}\left[f_{n}\left(\hat{X}_{n}^{\theta}\right)+i g_{n}\left(\hat{X}_{n}^{\theta^{\prime}}\right)\right]\right\rangle\right|^{2}
$$

This can be evaluated by the joint probability shown in Eq. (8.10), and will include terms like:

$$
\begin{aligned}
\left\langle f_{1}\left(\hat{X}_{1}^{\theta}\right) f_{2}\left(\hat{X}_{2}^{\phi}\right)\right\rangle= & \left(\frac{4}{\pi}\right) \iint e^{-2|\mathbf{X}|^{2}} f_{1} f_{2}\left[|\mathbf{X}|^{2}\right. \\
& \left.+2 X^{\theta} X^{\phi} \cos (\theta-\phi)\right] d^{2} \mathbf{X} .
\end{aligned}
$$

To show this more clearly, we give an example of the observables as fractional order moments $\left(\hat{X}^{\theta}\right)^{m}$ with corresponding outcomes $\left|x^{\theta}\right|^{m} \operatorname{sign}\left(x^{\theta}\right)$. Replacing $f_{1}$ and $f_{2}$ in the above integral with $\left(\hat{X}_{A}^{\theta}\right)^{m}$ and $\left(\hat{X}_{B}^{\theta}\right)^{m}$, we obtain that

$$
\begin{aligned}
& \left\langle\left(\hat{X}_{A}^{\theta}\right)^{\frac{1}{3}}\left(\hat{X}_{B}^{\phi}\right)^{\frac{1}{3}}\right\rangle=0.433881 \cos (\phi-\theta), \\
& \left\langle\left(\hat{X}_{A}^{\theta}\right)^{\frac{2}{3}}\left(\hat{X}_{B}^{\phi}\right)^{\frac{2}{3}}\right\rangle=0.42583
\end{aligned}
$$

for $m=1 / 3$ and $m=2 / 3$.

For convenience, we assume the functions $f_{n}$ and $g_{n}$ are odd, and thereby only the cross term in (8.13) has a nonzero contribution. The value of $L H S$ can be maxi- mized by using orthogonal angles so that $\cos (\theta-\phi)= \pm 1$ :

$$
\begin{aligned}
\text { LHS } & =\left|\left\langle\frac{\prod_{n=1}^{2}\left(f_{n}+g_{n}\right)+\prod_{n=1}^{2}\left(f_{n}-g_{n}\right)}{2}\right\rangle\right|^{2} \\
& =\left|\left(\frac{1}{\pi}\right)\left[\prod_{n=1}^{2} e^{-2 x^{2}} x f_{n}^{+} d x+\prod_{n=1}^{2} e^{-2 x^{2}} x f_{n}^{-} d x\right]\right|^{2} \\
& =\left|\left(\frac{1}{\pi}\right)\left[\prod_{n=1}^{2} I_{n}^{+}+\prod_{n=1}^{2} I_{n}^{-}\right]\right|^{2},
\end{aligned}
$$

where $f_{n}^{ \pm}=f_{n} \pm g_{n}$, and define integrals for $x: I_{n}^{ \pm}=$ $2 \int e^{-2 x^{2}} x f_{n}^{ \pm} d x$.

While the value of RHS

$$
\begin{aligned}
R H S & =\left\langle\prod_{n=1}^{2}\left[f_{n}\left(\hat{X}_{n}^{\theta}\right)^{2}+g_{n}\left(\hat{X}_{n}^{\theta^{\prime}}\right)^{2}\right]\right\rangle \\
& =\left\langle\prod_{n=1}^{2} \frac{\left(\hat{f}_{n}+\hat{g}_{n}\right)^{2}+\left(\hat{f}_{n}-\hat{g}_{n}\right)^{2}}{2}\right\rangle
\end{aligned}
$$

is invariant with angles as it only related to the square items in the probability of Eq. (8.10). Define $I_{n}=$ $\left.4 \int x^{2} e^{-2 x^{2}}\left[\left(f_{n}^{+}\right)^{2}+\left(f_{n}^{-}\right)^{2}\right)\right] d x$, and $I_{n}^{o}=\int e^{-2 x^{2}}\left[\left(f_{n}^{+}\right)^{2}+\right.$ $\left.\left.\left(f_{n}^{-}\right)^{2}\right)\right] d x$, then we can obtain

$$
R H S=\frac{1}{4 \pi}\left[\prod_{n=1}^{r} I_{n} \prod_{k=r+1}^{N} I_{n}^{o}+\prod_{n=1}^{r} I_{n}^{o} \prod_{k=r+1}^{N} I_{n}\right]
$$

where $r=1, N=2$.
[1] J. S. Bell, Physics 1, 195 (1964).

[2] J. F. Clauser and A. Shimony, Rep. Progr. Phys. 41, 1881 (1978).

[3] A. Zeilinger, Rev. Mod. Phys. 71, S288 (1999).

[4] A. Aspect, J. Dalibard, and G. Roger, Phys. Rev. Lett. 49, 1804 (1982).

[5] J. A. Larsson, Phys. Lett. A 256, 245 (1999); Anupam Garg and N. D. Mermin, Phys. Rev. D 35, 3831 (1987).

[6] M. A. Rowe, D. Kielpinski, V. Meyer, C. A. Sackett, W. M. Itano, C. Monroe, and D. J. Wineland, Nature 66, 033802 (2001).

[7] G. Weihs, T. Jennewein, C. Simon, H. Weinfurter, and A. Zeilinger, Phys. Rev. Lett. 81, 5039 (1998).

[8] U. Leonhardt and J. A. Vaccaro, J. Mod Opt, 42, 939 (1995); A. Gilchrist, P. Deuar, and M. D. Reid, Phys. Rev. Lett. 80, 3169 (1998); B. Yurke, M. Hillery, and D. Stoler, Phys. Rev. A 60, 3444 (1999). J. Wenger et al., ibid., 67, 012105 (2003).

[9] Z. Y. Ou, S. F. Pereira, H. J. Kimble, and K. C. Peng, Phys. Rev. Lett. 68, 3663 (1992).

[10] M. D. Reid, P. D. Drummond, W. P. Bowen, E. G. Cav- alcanti, P. K. Lam, H. A. Bachor, U. L. Andersen, G. Leuchs, Rev. Mod. Phys. 81, 1727 (2009).

[11] J. F. Clauser, M. A. Horne, A. Shimony, and R. A. Holt, Phys. Rev. Lett. 23, 880 (1969).

[12] J. Wenger, M. Hafezi, F. Grosshans, R. T. Brouri, and P. Grangier, Phys. Rev. A 67, 012105 (2003).

[13] H. Nha and H. J. Carmichael, Phys. Rev. Lett. 93, 020401 (2004).

[14] W. J. Munro and G. J. Milburn, Phys. Rev. Lett. 81, 4285 (1998); W. J. Munro, Phys Rev. A 59, 4197 (1999).

[15] A. Acin, N. J. Cerf, A. Ferraro, and J. Niset, Phys. Rev. A 79, 012112 (2009).

[16] N. D. Mermin, Physics Today, 42, 9 (1989); Phys. Rev. Lett. 65, 1838 (1990); M. Ardehali, Phys. Rev. A 46, 5375 (1992). D. M. Greenberger, M. A. Horne, and A. Zeilinger, in Bell's Theorem, Quantum Theory, and Conceptions of the Universe, edited by M. Kafatos (Kluwer Academic, Dordrecht, Holland, 1989), p. 69.

[17] D. N. Klyshko, Phys. Lett. A 172, 399 (1993); A. V. Belinskii and D. N. Klyshko, Phys. Usp. 36, 653 (1993).

[18] E. G. Cavalcanti, C. J. Foster, M. D. Reid, and P. D. 
Drummond, Phys. Rev. Lett. 99, 210405 (2007).

[19] E. Shchukin and W. Vogel, Phys. Rev. A 78, 032104 (2008).

[20] A. Salles, D. Cavalcanti, A. Acin, Phys. Rev. Lett. 101, 040404 (2008).

[21] Alejo Salles, Daniel Cavalcanti, Antonio Acin, David Perez-Garcia, and Michael M. Wolf, arXiv:1002.1893 (2010).

[22] Q. Y. He, E. G. Cavalcanti, M. D. Reid, and P. D. Drummond, Phys Rev Lett. 103, 180402 (2009).

[23] Bearing in mind these measurements can be noncompatible, the LHS is simply an abbreviation for the expansion in terms of observable moments, eg $\left\{\left\langle x_{1} x_{2}\right\rangle-\right.$ $\left.\left\langle p_{1} p_{2}\right\rangle\right\}^{2}+\left\{\left\langle x_{1} p_{2}\right\rangle+\left\langle x_{2} p_{1}\right\rangle\right\}^{2}$ when $N=2$.

[24] S. L. Braunstein and A. Mann. Phys. Rev. A 47, R2427 (1993); S. S. Jang, Y. W. Cheong, J. Kim, and H. W. Lee, Phys. Rev. A 74, 062112 (2006).

[25] A. Cabello, D. Rodriguez and I. Villanueva, Phys. Rev. Lett. 101, 120402 (2008).

[26] W. Dur, G. Vidal, and J. I. Cirac, Phys. Rev. A 62, 062314 (2000).
[27] R. Raussendorf and H. J. Briegel, Phys. Rev. Lett. 86, 5188 (2001); R. Raussendorf, D. E. Browne, and H. J. Briegel, Phys. Rev. A 68, 022312, (2003).

[28] C. A. Sackett et al., Nature (London) 404, 256 (2000); Z. Zhao et al., Phys. Rev. Lett. 91, 180401 (2003); Z. Zhao et al., Nature (London) 430, 54 (2004); D. Leibfried et al., Nature (London) 438, 639 (2005).

[29] P. Walther et al, Nature 434, 169 (2005). P. Walther et al, Phys. Rev. Lett. 95, 020403 (2005).

[30] C. Y. Lu et al, Nature Physics 3, 91 (2007).

[31] Sliwa and Banaszek, Phys. Rev. A 67, 030101(R) (2003).

[32] V. Scarani, A. Acin, E Schenck, and M. Aspelmeyer, Phys. Rev. A 71, 042325 (2005).

[33] H. M. Wiseman, S. J. Jones, and A. C. Doherty, Phys. Rev. Lett. 98, 140402 (2007); S. J. Jones, H. M. Wiseman, and A. C. Doherty, Physical Review A 76, 052116 (2007); E. G. Cavalcanti, S. J. Jones, H. M. Wiseman, and M. D. Reid, Phys Rev A 80, 032112 (2009).

[34] W. H. Louisell, "Quantum statistical properties of radiation" (Wiley, New York, 1973). 